\title{
A General Bargaining Model of Legislative Policy-making
}

\author{
Jeffrey S. Banks ${ }^{1}$ and John Duggan*, ${ }^{*}, 2$ \\ ${ }^{1}$ Division of Humanities and Social Sciences, California Institute of Technology, Pasadena, \\ CA 91125 \\ ${ }^{2}$ Department of Political Science and Department of Economics, University of Rochester, \\ Rochester, NY 14627
}

\begin{abstract}
We present a general model of legislative bargaining in which the status quo is an arbitrary point in a multidimensional policy space. In contrast to other bargaining models, the status quo is not assumed to be bad for all legislators, and delay may be Pareto efficient. We prove existence of stationary equilibria. We show that if all legislators are risk averse or if even limited transfers are possible, then delay is only possible if the status quo lies in the core. Thus, we expect immediate agreement in multidimensional models, where the core is typically empty. In one dimension, delay is possible if and only if the status quo lies in the core of the voting rule, and then it is the only possible outcome. Our comparative statics analysis yield two noteworthy insights: moderate status quos imply moderate policy outcomes, and legislative patience implies policy moderation.
\end{abstract}

A central objective of research in political economy is to obtain a general understanding of the determinants of public policy in a majoritarian society. Black's (1958) median voter theorem provides compelling predictions when policies are restricted to a single dimension, but the theory of social choice has yielded quite negative results

* This paper was completed after Jeff Banks's death. I am deeply indebted to him for his friendship and his collaboration on this and many other projects.

+ Support from the National Science Foundation, grant numbers SES-9975173 and SES-0213738, is gratefully acknowledged.

MS submitted 16 March 2005; final version received 14 fune 2005

ISSN 1554-0626; DOI 10.1561/100.00000001

(C) 2006 now Publishers. 
on the existence of majority rule equilibria in multiple dimensions. ${ }^{1}$ A tool of potential importance in surmounting this obstacle is the non-cooperative theory of bargaining, deriving from the work of Rubinstein (1982). The theory has found numerous applications, including distributive politics (Baron and Ferejohn 1989), government formation (Merlo 1997; Diermeier, Eraslan, and Merlo 2002), international relations (Powell 2002), and bankruptcy (Eraslan 2002a). Applications to policy-making in a legislative body, however, are few in number and limited by the implicit assumption, ubiquitous in the game-theoretic literature on bargaining, that failure to reach an agreement is worse for all legislators than every possible bargaining outcome.

This assumption of a bad status quo can be traced to Rubinstein's (1982) model, where two people must agree on a division of a resource and take turns making offers and responding to offers. In the simplest version of this model, a legislator's payoff is just the share of resource consumed, and therefore the payoff in any period an agreement is not reached is simply zero: the status quo in this model is no consumption, which is obviously worse for the legislators than any division the two might decide on. Binmore (1987) elaborates on the basic framework by allowing the proposer to be randomly selected in each period. In a seminal paper, Baron and Ferejohn (1989) apply this approach to model legislative allocation of pork barrel goods, assuming any finite number of legislators and voting by majority rule: in their closed-rule model, a legislator is randomly selected to propose an allocation of spending across districts; if this proposal is passed by a majority of the legislature, then the game ends with that allocation; otherwise, the game continues to the next period, where this process is repeated. Legislators do not enjoy the benefits of pork until it is allocated and so, as in the basic model, the status quo is zero. The theory of bargaining in distributive settings is now well developed, ${ }^{2}$ but the application to pork barrel spending misses the public good aspect of public policy and the fact that "more" is not always preferred to "less."

The first application to public policy is due to Baron (1991), who considers examples of policy-making in a two-dimensional issue space, assuming three and four legislators and quadratic utilities. Thus, each legislator has circular indifference curves centered around a unique ideal point in the policy space. Legislators are assumed to be perfectly patient, however, so that delay is costless and considerations of the status quo are moot. Jackson and Moselle (2002) assume a unidimensional policy space in addition to a divisible resource, and Banks and Duggan (2000) generalize the above models by simply allowing any convex set of policies and concave utilities. There, we prove existence of stationary equilibria in multidimensional issue spaces and, for the case of one dimension, we show that the equilibrium outcomes of bargaining converge to the ideal point of the median voter as the legislators place greater weight on the future. These models of policy-making,

1 See Plott (1967), Rubinstein (1979), Schofield (1983), Cox (1984), Le Breton (1987), McKelvey and Schofield (1987), Banks (1995), and Saari (1997).

2 Theoretical analyses building on this work have studied risk aversion (Harrington 1989, 1990a, 1990b), externalities in consumption (Calvert and Dietz 2005), veto rules (Winter 1996, McCarty 2000), a stochastic resource constraint (Merlo and Wilson 1995, Eraslan and Merlo 2002), and uniqueness of stationary equilibria (Eraslan 2002b, Cho and Duggan 2003). 
except for Baron (1991) who imposes perfect patience, follow the above-cited papers in assuming a bad status quo. But while that assumption may be reasonable in the context of distributing pork, it is less desirable in a bargaining model of policy-making, where it is likely that at least some legislators are happy with the status quo - and it is very unlikely that every legislator prefers every policy to the status quo.

In this paper we model the status quo as an arbitrary, perhaps Pareto-efficient, policy. We follow Banks and Duggan (2000) by allowing for a general multidimensional issue space and general utility functions on the part of legislators. We assume the proposer in any period is randomly selected, as in Baron and Ferejohn (1989), but we allow for an arbitrary voting rule. We capture unanimity rule, majority rule, and veto rules as special cases, and we even allow the legislators to be partitioned into two houses, a majority in each required for passage. Our model generates insights into some well-known special cases, including Romer and Rosenthal's (1978a,1978b) agenda-setting model, in which a fixed proposer makes a take-it-or-leave-it offer subject to a majority vote, as well as Krehbiel's $(1996,1998)$ model of a unicameral legislature with a filibuster rule and executive veto. We capture these one-shot models by assuming impatient legislators (setting discount factors equal to zero) and a single issue dimension, but our general framework allows us to investigate the effects of adding an infinite-horizon (positive discount factors) and more issue dimensions. Similarly, imbedding the model of Baron and Ferejohn (1989) in our framework, we can consider the effects of changing the status quo from zero to, for example, equal division. We show, for example, that adding an infinite horizon to the Romer-Rosenthal or Krehbiel models does not affect their results when utilities are quadratic, while a positive status quo in the Baron-Ferejohn model reduces the rents of the proposer.

Our first general result is that, despite the fact that some legislators may favor the status quo, there always exists a stationary equilibrium in which every proposal made passes, i.e., there exists a no-delay stationary equilibrium. Furthermore, we prove that the nodelay stationary equilibrium proposals are upper hemicontinuous in the parameters of the model: small perturbations of the parameters cannot produce equilibria far from those at the original specification. This is illustrated in Romer and Rosenthal's (1978a,1978b) agenda-setting model, where the setter's proposal varies continuously with voter ideal points and the status quo. An implication is that, if we give voters a small amount of patience and give the agenda setter a proposal probability slightly less than one, then equilibrium outcomes will be close to Romer and Rosenthal's solution. And if we imbed their model in a multidimensional space and ideal points are close to collinear, then equilibrium outcomes will again be close to the original ones. Similar observations also hold for Krehbiel's $(1996,1998)$ legislative model. Finally, if we modify the Baron and Ferejohn (1989) model to allow for a status quo with slightly positive consumptions of the resource, or if we give the legislators a small amount of risk aversion, then the equilibrium outcomes will be close to theirs.

An advantage of our approach to modeling the status quo is that we can now more meaningfully consider the possibility of delay in bargaining, which, among the abovementioned models, was possible only with some probability of a relaxed resource 
constraint in the future (Merlo and Wilson 1995, Eraslan and Merlo 2002). ${ }^{3}$ We show that delay is possible in our model, but only under very specific circumstances. If all legislators are risk averse or if even limited transfers are possible, then delay is only possible if the status quo lies in the core, and in that case the only possible outcome once a decision is made is the status quo itself. Since the core, the set of policies undominated according to the voting rule, is almost always empty when the policy space is multidimensional, we conclude that there will typically be immediate agreement in multidimensional settings.

Our results are sharpest for unidimensional policy spaces. We show that when the status quo is in the core, the equilibrium outcome is the status quo every period. We refer to this phenomenon as "core selection," which is related to the notion of core equivalence in Banks and Duggan (2000). In contrast to the core equivalence results in that paper, which assume very patient legislators, here we make no assumption about the rate of time discounting by legislators. When voting is by majority rule, of course, it is known that the core consists of the median policies, and our core selection result yields a new game-theoretic perspective on Black's (1958) median voter theorem: whereas Black arrives at the median from a social choice theory approach, we give conditions under which strategic, forward-looking legislators arrive at the same outcome. We also show that, if the status quo is not in the core, then it is never proposed. Thus, occurrence of the status quo in equilibrium is all or nothing, i.e., it is always proposed by every legislator (if it is in the core) or never proposed by any legislator (if it is outside the core).

Finally, we present comparative static results on the patience of legislators and on the location of the status quo for the unidimensional model. First, as a corollary of our core selection and continuity results, if the status quo is close to the core, then the stationary equilibrium proposals of the legislators will also be close to the core. In other words, moderate status quos imply moderate policy outcomes. Second, if the legislators are sufficiently patient, then, regardless of the location of the status quo, all equilibrium proposals will be arbitrarily close to the core. That is, legislative patience implies policy moderation. The latter comparative static delivers an asymptotic version of the median voter theorem, one that is independent of the location of the status quo. It also suggests the following for the design of electoral institutions, which are unmodeled here: if we view the discount factor of a legislator as corresponding to the probability of reelection, then systems that offer a sufficient incumbency advantage will lead to moderate policies.

The rest of the paper is organized as follows. In Section 2, we present the model and formal definition of our equilibrium concept. In Section 3, we present a number of special cases, including the Romer and Rosenthal (1978a,b) agenda-setting model, Krehbiel's $(1996,1998)$ legislative bargaining model, and a generalization of the Baron and Ferejohn (1989) bargaining model with an arbitrary status quo. In Section 4, we present our results on equilibrium existence, stability of the status quo, and core selection. In Section 5, we conclude. An Appendix contains proofs of theorems and other technical considerations.

3 Delay may also be obtained by adding incomplete information to the model, as in Rubinstein (1985). 


\section{THE LEGISLATIVE BARGAINING MODEL}

Let $X \subseteq \Re^{d}$ denote a nonempty, compact, convex set of policies with at least two policy alternatives, and let $q \in X$ denote the status quo policy. Let $N=\{1, \ldots, n\}$ denote a set of legislators, with $n \geq 2$, who play an infinite-horizon bargaining game over the set of policies. The timing of interaction is as follows. If no policy has been accepted prior to period $t$, then (1) legislator $i$ is recognized with probability $\rho_{i} \geq 0$, where $\sum_{i \in N} \rho_{i}=1$; then (2) the selected legislator $i$ makes a proposal $p_{i} \in X$; then (3) every legislator $j \in N$ simultaneously votes to either accept or reject the proposal. The recognition probabilities, $\rho_{1}, \ldots, \rho_{n}$, are exogenously fixed throughout the game. Let $\mathcal{D} \subseteq 2^{N} \backslash\{\emptyset\}$ denote a collection of decisive coalitions, also exogenously fixed. ${ }^{4}$ If the group of legislators voting for the proposal is decisive, i.e., $\{j \in N \mid j$ accepts $\} \in \mathcal{D}$, then the proposal $p_{i}$ is the chosen policy and bargaining ends with outcome $p_{i}$ in period $t$ and in every subsequent period. Otherwise, the outcome in period $t$ is $q$, and steps $1-3$ are repeated for period $t+1$.

We impose on the voting rule $\mathcal{D}$ only the minimal conditions that it is nonempty and monotonic, in the sense that any superset of a decisive coalition is itself decisive: $C \in \mathcal{D}$ and $C \subseteq C^{\prime}$ imply $C^{\prime} \in \mathcal{D}$. Thus, we allow for a variety of voting rules beyond majority rule $(\mathcal{D}=\{C \subseteq N|| C \mid>n / 2\})$, such as unanimity rule $(\mathcal{D}=\{N\})$, and dictatorship $(\mathcal{D}=\{C \subseteq N \mid i \in C\}$ for some $i \in N)$. For another example, a proposal may require a majority of legislators other than a designated legislator, say $n$, to pass, i.e.,

$$
\mathcal{D}^{R R}=\left\{C \subseteq N|| C \backslash\{n\} \mid>\frac{n-1}{2}\right\} .
$$

Letting $\rho_{n}=1$, we have the set-up of the agenda-setting model of Romer and Rosenthal (1978a, 1978b), where legislator $n$ plays the role of agenda setter. Or we can capture a unicameral legislature and executive with a filibuster rule ( $3 / 5$ cloture rule) and executive veto $(2 / 3$ override rule) as follows. Letting $n$ denote the executive and $N \backslash\{n\}$ the legislature, define

$$
\begin{gathered}
\mathcal{D}^{K}=\{C \subseteq N \mid \\
|C \backslash\{n\}| \geq \frac{2(n-1)}{3} \text { or both } n \in C \\
\text { and } \left.|C \backslash\{n\}| \geq \frac{3(n-1)}{5}\right\},
\end{gathered}
$$

That is, a coalition is decisive if it contains two thirds of the legislature or three fifths plus the executive. Letting $m$ denote the median legislator and $\rho_{m}=1$, we have the set-up of the legislative model of Krehbiel $(1996,1998)$. Some of our results do use the assumption that $\mathcal{D}$ is proper, i.e., if $C \in \mathcal{D}$, then $N \backslash C \notin \mathcal{D}$. This is clearly a weak condition: when $\mathcal{D}$ is used to define strict social preferences, as in the theory of social choice, it is always satisfied.

4 In the terminology of cooperative game theory, $\mathcal{D}$ describes a "simple game," and the elements of $\mathcal{D}$ are "winning coalitions." 
Each legislator $i$ 's preferences over sequences of policy outcomes are described by a von Neumann-Morgenstern utility representation $u_{i}: X \rightarrow \Re$ and a common discount factor $\delta \in[0,1)$ as follows. Given a sequence $x_{1}, x_{2}, \ldots$ of policies over time, $i$ 's payoff is

$$
(1-\delta) \sum_{t=1}^{\infty} \delta^{t-1} u_{i}\left(x_{t}\right)
$$

Of course, the outcomes of the bargaining game defined above are of a simple form: either the status quo obtains in every period, in which case $i$ 's payoff is just $u_{i}(q)$, or some other proposal is passed in some period; in that case, $i$ receives payoff

$$
\left(1-\delta^{t-1}\right) u_{i}(q)+\delta^{t-1} u_{i}(x)
$$

if policy $x$ is proposed and accepted in period $t$.

We assume throughout that each $u_{i}$ is continuous and concave. In the Appendix, we define a further technical condition on utilities that is assumed throughout. The condition, called limited shared meak preference (LSWP), says that any policy $y$ that is weakly preferred to a policy $x$ by all members of a decisive coalition can be approximated by policies that all coalition members strictly prefer to $x$. An implication of LSWP, with our other conditions, is that each legislator $i$ has a unique ideal point, denoted $\tilde{x}^{i}$, that is strictly preferred to every other policy, i.e., $u_{i}\left(\tilde{x}^{i}\right)>u_{i}(y)$ for all $y \in X \backslash\left\{\tilde{x}^{i}\right\}$. Many familiar environments satisfy LSWP, including the following. ${ }^{5}$

- Classical spatial model/Pure public goods. Alternatives are vectors of ideological policies or public good levels. Each $u_{i}$ is strictly quasi-concave, as when $u_{i}(x)=-\left\|x-\tilde{x}^{i}\right\|$ or $u_{i}(x)=-\left\|x-\tilde{x}^{i}\right\|^{2}$.

- Public decisions with transfers. The policy space $X$ is a subset of $Z \times T$, where $Z$ is a space of public decisions and $T \subseteq \Re^{n}$ is a space of allocations of a district-specific good, and each $u_{i}$ is quasi-linear: $u_{i}(z, t)=\phi_{i}(z)+t_{i}$, with $\phi_{i}$ strictly quasi-concave.

- Divide the dollar. The policy space is $X=\left\{\left(x_{1}, \ldots, x_{n}\right) \in \Re_{+}^{n} \mid \sum_{i \in N} x_{i} \leq 1\right\}$, representing allocations of pork to legislative districts, and $u_{i}(x)$ is strictly increasing in $x_{i}$, constant in the consumption of other legislative districts.

- Local public goods. Alternatives are allocations of any number of local public goods to districts, where each $u_{i}$ is strictly quasi-concave and strictly monotonic in the consumption of $i$ 's district, constant in the consumption of other districts.

The more familiar condition of strict quasi-concavity is sufficient for LSWP, but it is not satisfied in the last three models, which we consider to be of potential importance for applications. The last model, in particular, generalizes divide-the-dollar environments but has not to our knowledge been considered in analyses of legislative policy-making. We therefore use the weaker LSWP condition.

Given a collection of decisive coalitions and legislator preferences, we define the core, denoted $K$, as the set of policies that cannot be overturned by any decisive coalition.

5 In Banks and Duggan (1999), we prove that LSWP holds in two general models, from which all of the examples below can be obtained as special cases. 
Formally,

$$
K=\left\{\begin{array}{l|l}
x \in X & \begin{array}{l}
\text { there do not exist } y \in X \text { and } C \in \mathcal{D} \\
\text { such that, for all } i \in C, u_{i}(y)>u_{i}(x)
\end{array}
\end{array}\right\} .
$$

For a familiar example of the core, let the voting rule $\mathcal{D}$ be unanimity rule, so that a policy $x$ is in the core if and only if there is no policy $y$ such that $u_{i}(y)>u_{i}(x)$ for every legislator $i \in N$, i.e., $x$ is weakly Pareto optimal. In this case, the core is nonempty and often quite large. When the set of policies is unidimensional and $\mathcal{D}$ is majority rule, it is known that the core is nonempty and consists of the median ideal point, or perhaps the interval between two median ideal points if the number of legislators is even. The core has a similar characterization in the general unidimensional case, as long as the voting rule is proper: it is nonempty and consists of the interval between two legislators' ideal points. ${ }^{6}$ In higher dimensional spaces, however, Plott (1967) and others have shown that the majority core points must satisfy conditions so restrictive that they would almost never be met in reality. Banks (1995) and Saari (1997) extend these results to other voting rules that require less than unanimity for passage. Thus, if there are many issue dimensions, the core is typically empty for such voting rules.

Complete information of preferences, the structure of the game form, etc., is assumed throughout. A history of length $t$ in the bargaining game describes all that has transpired in the first $t$ periods (who the previous proposers were, what they proposed, how legislators voted), and a strategy for a legislator is a mapping specifying an intended action (what to propose, how to vote) as a function of all histories of all lengths. Since our focus in this paper is only on equilibria in stationary strategies, we provide a formal definition only of such strategies. A (pure) stationary strategy for legislator $i$ consists of a proposal $p_{i} \in X$, to be offered anytime $i$ is recognized, and a measurable voting rule $r_{i}: X \rightarrow$ \{accept, reject\}. For the latter, we will use the more convenient representation of an acceptance set, $A_{i}=r_{i}^{-1}$ (accept), i.e., the set of proposals that $i$ would vote to accept. Given a profile $\left(A_{1}, \ldots, A_{n}\right)$ of acceptance sets and given $C \subseteq N$, define the set

$$
A_{C}=\bigcap_{i \in C} A_{i}
$$

of proposals acceptable to all members of $C$, and define the social acceptance set

$$
A=\bigcup_{C \in \mathcal{D}} A_{C},
$$

consisting of proposals that could be passed in any and all periods.

It turns out that mixtures over proposals are required for our most general existence result, so let $\mathcal{P}(X)$ denote the set of probability distributions on $X$ and endow $\mathcal{P}(X)$ with the topology of weak convergence. ${ }^{7}$ Given $Y \subseteq X$, let $\mathcal{P}(Y)$ denote the subset of

6 See the Appendix for a formal statement.

7 Formally, $\mathcal{P}(X)$ is the set of Borel probability measures on $X$. A sequence $\left\{\pi^{k}\right\}$ of probability measures converges meakly to $\pi$ if and only if, for every (bounded) continuous function $f: X \rightarrow \Re$, we have $\int f d \pi^{k} \rightarrow \int f d \pi$ 
probability distributions on $X$ that place probability one on $Y$. Let $\pi_{i} \in \mathcal{P}(X)$ denote a mixed stationary proposal for legislator $i$, where $\pi_{i}(Y)$ denotes the probability that $i$ proposes an alternative in the set $Y$, and let $\pi=\left(\pi_{1}, \ldots, \pi_{n}\right)$ denote a profile of mixed stationary proposals. A mixed stationary strategy for $i$ is a pair $\sigma_{i}=\left(\pi_{i}, A_{i}\right)$, and we let $\sigma=\left(\sigma_{1}, \ldots, \sigma_{n}\right)$ denote a profile of mixed stationary strategies. It is important to note that any randomization over proposals takes place before voting: the legislators know which policy has been proposed before they cast their votes.

Informally, a profile $\sigma$ constitutes a stationary equilibrium if, for every legislator $i \in N$, the proposal strategy $\pi_{i}$ is optimal given the acceptance sets $\left(A_{1}, \ldots, A_{n}\right)$ of the other legislators, and the acceptance set $A_{i}$ is optimal given that $\sigma$ describes what would happen if the current proposal were rejected. To formalize these conditions, note first that any strategy profile $\sigma$ defines in an obvious (if notationally dense) manner a probability distribution over sequences of outcomes and, with it, an expected utility $v_{i}(\sigma)$ for each legislator $i \in N$ as evaluated at the beginning of the game. By stationarity, this is also $i$ 's continuation value throughout the game, i.e., $i$ 's expected utility evaluated at the beginning of next period if the current period's proposal is rejected.

Formally, $\sigma$ is a stationary equilibrium if two conditions hold. First, we require that the legislators' acceptance sets satisfy meak dominance, i.e., legislator $i$ votes for proposal $x$ if and only if the utility from $x$ is at least that of rejecting the proposal and continuing to the next period. ${ }^{8}$ That is, for all $i \in N$, we require that

$$
A_{i}=\left\{x \in X \mid u_{i}(x) \geq(1-\delta) u_{i}(q)+\delta v_{i}(\sigma)\right\} .
$$

This condition eliminates implausible equilibria in which, for instance, under majority rule everyone accepts every proposal independently of preferences: the problem in such situations is that no one's vote will change the outcome of the game, and hence everyone's vote is a best response, despite the fact that some legislators may be voting for undesirable policies. Note that weak dominance implies $q \in A_{i}$ if $\delta=0$. Second, we require that the legislators' proposals satisfy sequential rationality, i.e., legislator $i$, when recognized as proposer, either chooses utility-maximizing outcomes from within $A$ or chooses an outcome that will be rejected, depending on which yields the higher payoff. That is, for all $i \in N$, we require that

$$
\pi_{i}\left(\arg \max \left\{u_{i}(y) \mid y \in A\right\}\right)=1
$$

when $\sup \left\{u_{i}(y) \mid y \in A\right\}>(1-\delta) u_{i}(q)+\delta v_{i}(\sigma)$; that $\pi_{i}(X \backslash A)=1$ when the inequality is reversed; and that $\pi_{i}$ place probability one on the union of these two sets when equality holds.

The explicit formula for legislator $i$ 's continuation value, given stationary strategy profile $\sigma$, is straightforward to derive: it is

$$
v_{i}(\sigma)=\frac{\sum_{j \in N} \rho_{j}\left[\int_{A} u_{i}(z) \pi_{j}(d z)+(1-\delta) \pi_{j}(X \backslash A) u_{i}(q)\right]}{1-\delta \sum_{j \in N} \rho_{j} \pi_{j}(X \backslash A)} .
$$

8 Baron and Kalai (1993) refer to such strategies as "stage-undominated." 
Since the discount factor $\delta$ is identical across legislators, we may write each $v_{i}(\sigma)$ as the expectation of $u_{i}$ with respect to a single probability distribution, say $v$, independent of $i$. This is explicitly defined in the Appendix. Let $\gamma$ be the probability distribution on $X$ that selects the status quo $q$ with probability $(1-\delta)$ and with probability $\delta$ draws an alternative from the distribution $v$. Then the expectation of $u_{i}$ with respect to $\gamma$ is just $(1-\delta) u_{i}(q)+\delta v_{i}(\sigma)$, and weak dominance can be written as

$$
A_{i}=\left\{x \in X \mid u_{i}(x) \geq \int u_{i}(z) \gamma(d z)\right\} .
$$

That is, legislator $i$ votes for proposals with utility at least equal to $i$ 's expected utility under $\gamma$. Because $\gamma$ contains all of the relevant information for any legislator to evaluate any proposed policy, we refer to $\gamma$ as the continuation distribution corresponding to $\sigma$. Letting $x(\gamma)$ denote the mean of the continuation distribution, concavity of $u_{i}$ implies $u_{i}(x(\gamma)) \geq \int u_{i} d \gamma$. We conclude that, given any profile $\sigma$ satisfying weak dominance, $x(\gamma) \in A_{i}$ for every legislator $i \in N$. In particular, the social acceptance set $A$ will be nonempty for such profiles.

A stationary equilibrium with delay is a profile $\sigma$ in which, with some positive probability, a proposal is rejected in the first (or any) period. Formally, this is $\sum_{i \in N} \rho_{i} \pi_{i}(A)<1$. A no-delay stationary equilibrium is then a profile such that each legislator proposes only policies in the acceptance set, i.e., $\sum_{i \in N} \rho_{i} \pi_{i}(A)=1$. Note that the status quo may be the outcome of a no-delay equilibrium, if it is in the social acceptance set and it is proposed with positive probability, i.e., if $q \in A$ and $\pi_{i}(\{q\})>0$ for some legislator $i$. In a no-delay equilibrium, $i$ 's continuation value takes the especially simple form,

$$
v_{i}(\sigma)=\sum_{j \in N} \rho_{j} \int u_{i}(z) \pi_{j}(d z)
$$

where the terms in (1) multiplied by $\pi_{i}(X \backslash A)$ disappear, as they correspond to outcomes following rejected proposals.

A static stationary equilibrium is one in which the status quo is maintained with probability one, either because no policy is ever passed or because the status quo is the only policy passed with positive probability. Formally, these are profiles $\sigma$ such that $\sum_{i \in N} \rho_{i} \pi_{i}(A \backslash\{q\})=0$. Such equilibria might exhibit delay or might not, as when the status quo is socially acceptable and all legislators propose it.

\section{SPECIAL CASES OF THE MODEL}

In this section, we survey some special cases of the bargaining model and provide explicit solutions for stationary equilibria. Models 1, 2, and 5 imbed well-known models in the infinite-horizon framework with an arbitrary status quo. Other special cases extend the theory to new environments, such as the unidimensional Model 3, where the comparison between the equilibria of our model and the predictions of the median voter theorem are 
particularly interesting. While interesting applications in their own right, these examples will motivate many of the theoretical results to follow, and they will serve to demonstrate the limits of those results as well.

Model 1: The Romer-Rosenthal model. Let the agenda setter be $n$ with $\rho_{n}=1$, let the number $n-1$ of voters be odd, let $X \subseteq \Re$, let the voting rule be $\mathcal{D}^{R R}$, and let each $u_{i}$ be quadratic with ideal point $\tilde{x}_{i}$, i.e., $u_{i}(x)=-\left|x-\tilde{x}_{i}\right|^{2}$. Let legislator $m$ have the median ideal point among $N \backslash\{n\}$, and assume $\tilde{x}_{m}<\tilde{x}_{n}$. Though Romer and Rosenthal (1978a, 1978 b) analyze a static model, i.e., $\delta=0$, we allow any $\delta<1$ here. By Lemma 1 of Banks and Duggan (2005a), the assumption of quadratic utilities implies that legislator $m$ is decisive: $x \in A$ if and only if $u_{m}(x) \geq(1-\delta) u_{m}(q)+\delta v_{m}(\sigma)$ in any no-delay stationary equilibrium. Thus, legislator $n$ 's proposal $p_{n}$ must satisfy

$$
u_{m}\left(p_{n}\right) \geq(1-\delta) u_{m}(q)+\delta v_{m}(\sigma)
$$

in any no-delay equilibrium. Assuming $q$ is close enough to $\tilde{x}_{m}$, namely $\left|q-\tilde{x}_{m}\right|<$ $\left|\tilde{x}_{n}-\tilde{x}_{m}\right|$, this constraint will be binding: otherwise, since $u_{m}\left(p_{n}\right)=v_{m}(\sigma)$ in equilibrium, we would have $u_{m}\left(p_{n}\right)>u_{m}(q)$, and the agenda setter would do better to propose slightly to the right of $p_{n}$, contradicting sequential rationality. Thus, when the status quo is close to the median legislator, the proposal constraint (3) is indeed binding. Since $u_{m}\left(p_{n}\right)=v_{m}(\sigma)$ in equilibrium, we must therefore have $u_{m}\left(p_{n}\right)=u_{m}(q)$. We conclude that the unique no-delay stationary equilibrium, independent of the discount factor, is such that $p_{n}=\tilde{x}_{m}+\left|q-\tilde{x}_{m}\right|$, as in Romer and Rosenthal's static model.

In the above model, the status quo is proposed by the agenda setter if and only if it coincides with the core of the voting rule $\mathcal{D}^{R R}$, i.e., $q=\tilde{x}_{m}$. In this case, the core point is the unique policy outcome. And when the status quo is close to the core, the agenda setter $n$ 's optimal proposal is close to the core as well. Note that we used the assumption of quadratic utilities to derive the same solution in our infinite-horizon model as did Romer and Rosenthal (1979a, 1979b) in their static model. If that assumption is relaxed, the models will generally have different solutions.

Model 2: $\quad$ The Krehbiel model. Let $n$ be the executive, let the number $n-1$ of legislators be odd, let $X=[0,1]$, let the voting rule be $\mathcal{D}^{K}$, and let each $u_{i}$ be quadratic with ideal point $\tilde{x}_{i}$. Let legislator $m$ have the median ideal point among $N \backslash\{n\}$, and let $\rho_{m}=1$. Though Krehbiel $(1996,1998)$ analyzes a static model, i.e., $\delta=0$, we allow any $\delta<1$ here. Assuming the executive's ideal point is greater than those of the legislators, index the legislators in increasing order of their ideal points: $\tilde{x}_{1}<\tilde{x}_{2}<\cdots<\tilde{x}_{n}$. Ignoring integer problems, identify the legislators $v=2(n-1) / 3$ (the veto pivot) and $f=2(n-1) / 5$ (the filibuster pivot). It can be checked that the core of the voting rule $\mathcal{D}^{K}$ is just $\left[\tilde{x}_{f}, \tilde{x}_{v}\right]$. In any no-delay stationary equilibrium $\sigma$, legislator $i$ votes for proposal $x$ if and only if $u_{i}(x) \geq(1-\delta) u_{i}(q)+\delta v_{i}(\sigma)$. Letting $p_{m}$ denote legislator $m$ 's equilibrium proposal, we argue that it must take the following form. First, we claim that, if $q<\tilde{x}_{f}$, then

$$
p_{m}=\min \left\{2 \tilde{x}_{f}-q, \tilde{x}_{m}\right\} .
$$


To see why, note that sequential rationality trivially implies $q \leq p_{m}$. Furthermore, $v_{i}(\sigma)=u_{i}\left(p_{m}\right)$ for every legislator $i \in N$ in any no-delay stationary equilibrium, so $i$ votes for $p_{m}$ in equilibrium if and only if $u_{i}\left(p_{m}\right) \geq u_{i}(q)$. Then we must have $u_{f}\left(p_{m}\right) \geq u_{f}(q)$, for otherwise the proposal would not receive the vote of the filibuster pivot $f$ or any legislator $i \leq f$, so less than three fifths of legislators would vote to accept, and the proposal would fail, a contradiction. An implication is that $p_{m} \leq 2 \tilde{x}_{f}-q$. That $p_{m} \leq \tilde{x}_{m}$ follows from sequential rationality: if $\tilde{x}_{m}<p_{m} \leq 2 \tilde{x}_{f}-q$, then the median legislator could propose slightly to the left of $p_{m}$, this proposal would still gain the support of every legislator $i \geq f$ and still pass, ${ }^{9}$ improving the outcome for $m$ and contradicting sequential rationality. Thus, $p_{m}$ is no greater than $2 \tilde{x}_{f}-q$ or $\tilde{x}_{m}$. If $p_{m}$ is strictly less than the minimum of these two quantities, i.e., $p_{m}<\min \left\{2 \tilde{x}_{f}-q, \tilde{x}_{m}\right\}$, then the median legislator would do better to propose slightly to the right of $p_{m}$, again contradicting sequential rationality. This establishes the claim. Second, if $q \in\left[\tilde{x}_{f}, \tilde{x}_{v}\right]$, then we can show that $p_{m}=q$. Last, if $\tilde{x}_{v}<q$, then we have

$$
p_{m}=\max \left\{2 \tilde{x}_{v}-q, \tilde{x}_{m}\right\} .
$$

In fact, this is the solution derived by Krehbiel in his static model. Note that, as the status quo moves from zero to $\tilde{x}_{f}, p_{m}$ moves from the median toward $\tilde{x}_{f}$; for $q \in\left[\tilde{x}_{f}, \tilde{x}_{v}\right], p_{m}$ equals the status quo; and as the status quo moves from $\tilde{x}_{v}$ to one, $p_{m}$ moves continuously away from $\tilde{x}_{v}$ and toward $\tilde{x}_{m}$.

In the above model, the status quo is not proposed by the median legislator unless it is in the core of the voting rule $\mathcal{D}^{K}$. If the status quo does lie in the core, the interval $\left[\tilde{x}_{f}, \tilde{x}_{v}\right]$, then it is the median legislator's unique optimal proposal and the unique bargaining outcome in every period. This can certainly occur while the status quo is distinct from the median legislator's ideal point, i.e., $q \neq \tilde{x}_{m}$, and in that case a majority of legislators would in fact prefer the median ideal point to the bargaining outcome, a phenomenon referred to as "gridlock" by Krehbiel $(1996,1998)$. The above analysis shows that gridlock either does not occur (a policy other than the status quo is proposed by the median legislator and passes) or is complete (the status quo obtains in every period). Finally, note that when the status quo is close to the core, the median legislator's optimal proposal is close to the core as well.

The next model gives our first example of the bargaining approach applied to the case of a unidimensional policy space and majority rule.

Model 3: One dimension, majority rule. Let $n$ be odd, let $X=[0,1]$, let each $\rho_{i}=1 / n$, let $\mathcal{D}$ be majority rule, and let each $u_{i}$ be quadratic with ideal point $\tilde{x}_{i}$. Assume $\tilde{x}_{1}<$ $\tilde{x}_{2}<\cdots<\tilde{x}_{n}$, and let $q \leq \tilde{x}_{m}$, where $m=(n+1) / 2$ is the median legislator. We look for a no-delay stationary equilibrium of the following form: $p_{i}=\tilde{x}_{m}-\Delta$ for all $i<m$, $p_{m}=\tilde{x}_{m}$, and $p_{i}=\tilde{x}_{i}+\Delta$ for all $i>m$, where $\Delta>0$ is fixed. Let each $A_{i}$ consist of

$9 \quad$ The argument that all legislators $i \geq f$ to the right of the filibuster pivot would vote for the new proposal uses the fact that, with quadratic utilities, legislator preferences over lotteries are order restricted. This is proved by Cho and Duggan (2003). 
the policies that give legislator $i$ utility at least $v_{i}=(1-\delta) u_{i}(q)+(\delta / n) \sum_{j=1}^{n} u_{i}\left(p_{j}\right)$. By construction, therefore, weak dominance will be satisfied. We then need only find a value of $\Delta$ consistent with sequential rationality. By Lemma 1 of Banks and Duggan (2005a), legislator $m$ is decisive: $x \in A$ if and only if $u_{m}(x) \geq(1-\delta) u_{m}(q)+\delta v_{m}$. So let $\Delta$ satisfy

$$
u_{m}\left(\tilde{x}_{m}-\Delta\right)=(1-\delta) u_{m}(q)+\frac{\delta}{n}\left[\sum_{i<m} u_{m}\left(\tilde{x}_{m}-\Delta\right)+u_{m}\left(\tilde{x}_{m}\right)+\sum_{i>m} u_{m}\left(\tilde{x}_{m}+\Delta\right)\right],
$$

or, equivalently,

$$
\Delta^{2}=(1-\delta)\left(\tilde{x}_{m}-q\right)^{2}+\frac{\delta(n-1)}{n} \Delta^{2} .
$$

Solving, we find that

$$
\Delta=\sqrt{\frac{(1-\delta)\left(\tilde{x}_{m}-q\right)^{2}}{1-\delta(n-1) / n}} .
$$

For $\delta$ close enough to one or for $q$ close enough to $\tilde{x}_{m}$, we have $\tilde{x}_{i}<p_{i}=\min A$ for all $i<m$ and $\max A=p_{i}<\tilde{x}_{i}$ for all $i>m$, delivering sequential rationality. Note the logic of this equilibrium: by strict concavity, the utility from the policy $(1-\delta) q+\delta \tilde{x}_{m}$ is strictly greater than the continuation value $v_{i}$ for each legislator $i$; this gives any legislator $i$, when selected as proposer, a wedge to pull the policy outcome toward $i$ 's ideal point by a small amount. When $q=\tilde{x}_{m}$, we have $\Delta=0$, so that every legislator proposes the median and it is the outcome with probability one. It would then also be an equilibrium for every legislator to propose zero while keeping the same acceptance sets as in the latter equilibrium: then every proposal will be rejected, so the equilibrium exhibits delay, and the median is still the outcome with probability one.

In the above model, we have found a stationary equilibrium in pure strategies, and the equilibrium is no-delay. Furthermore, the core point, $\tilde{x}_{m}$, is proposed and passed with positive probability, but other proposals may be passed as well. Finally, the social acceptance set collapses to the core as legislators become arbitrarily patient or as the status quo converges to the core: $\Delta \rightarrow 0$ as $\delta \rightarrow 1$ or as $q \rightarrow \tilde{x}_{m}$. This illustrates the general claims made above that legislative patience leads to moderate outcomes, as do moderate status quos. Note also that when the status quo coincides with the legislative median, i.e., $q=\tilde{x}_{m}$, the social acceptance set collapses to this point, and the core policy is selected with probability one. Otherwise, when $q \neq \tilde{x}_{m}$, the status quo is the outcome with probability zero. Thus, as in the Romer-Rosenthal and Krehbiel models, the status quo is an all or nothing proposition.

Model 4: Tomo dimensions, core point exists. Let $n=5, X=[-1,1] \times[-1,1]$, let each $\rho_{i}=1 / 5$, let $q=(0,0)$, let $\mathcal{D}$ be majority rule, and let $u_{i}(x)=-\left\|x-\tilde{x}^{i}\right\|^{\lambda}$, with $\lambda \geq 1$ and ideal points at $(0,0),(1,0),(-1,0),(0,1)$, and $(0,-1)$. That is, the legislators' ideal points are at the center and four corners of a square, and indifference curves are perfect 
circles centered around them. Of course, the unique core point is $(0,0)$. We look for a no-delay stationary equilibrium of the form $p_{i}=(1-\alpha) \tilde{x}^{i}$ for each legislator $i$, where $\alpha \in[0,1]$, and $i$ 's proposal is accepted by the legislators at the two adjacent corners of the square. In such an equilibrium, legislator $i$ with $\tilde{x}^{i} \neq(0,0)$ would have continuation value

$$
v_{i}=-\frac{\delta}{5}\left[\alpha^{\lambda}+1+2\left(2-2 \alpha+\alpha^{2}\right)^{\lambda}+(2-\alpha)^{\lambda}\right] .
$$

Letting $A_{i}$ consist of the policies $x$ satisfying $\left\|x-\tilde{x}^{i}\right\|^{\lambda} \leq(1-\delta)-\delta v_{i}$, weak dominance will be satisfied. We then need only specify a value of $\alpha$ consistent with sequential rationality. We do so for the values $\lambda=2,4$. When $\lambda=2$, it is straightforward to verify that $\alpha=1$, i.e., $p_{i}=(0,0)$ for every legislator $i \in N$, yields a stationary equilibrium: in this case, each legislator's continuation value is -1 and each accepts a proposal only if it yields a utility at least that of the core point; then the optimal proposal is indeed $(0,0)$. When $\lambda=4$, this is still an equilibrium. It can be checked that $\alpha=0$, meaning that all legislators propose their own ideal points, is also an equilibrium, as long as $\delta \geq 3 / 4$. Consider the proposal of the legislator with ideal point $(1,0)$. That legislator proposes $(1,0)$, which gives the adjacent legislators utility -4 . Their continuation values are $-5 \delta$. So given our specification of acceptance sets, they accept $(1,0)$ if $4 \leq 1+4 \delta$, which holds if $\delta \geq 3 / 4$. Thus, $(1,0)$ is accepted by a majority and is that legislator's optimal proposal, and similarly for the other legislators.

In the above model, regardless of $\lambda$, the status quo is proposed by the legislator with ideal point at the status quo, which is in the core. In contrast to the $q=\tilde{x}_{m}$ case from Model 3, there may be no-delay equilibria in which other legislators propose other policies, even though the status quo is in the core. In contrast with all of the preceding models, in the next the core is empty.

Model 5: The Baron and Ferejohn model with arbitrary status quo. Let $n$ be odd, let $X=\left\{\left(x_{1}, \ldots, x_{n}\right) \in \Re_{+}^{n} \mid \sum_{i \in N} x_{i} \leq 1\right\}$ be the unit simplex in $\Re^{n}$, let $\mathcal{D}$ be majority rule, and let $u_{i}\left(x_{1}, \ldots, x_{n}\right)=x_{i}$. We generalize Baron and Ferejohn (1989) by allowing for an arbitrary $q \in X$, whereas they assume $q=0$. We will look for a no-delay stationary equilibrium such that each legislator offers a positive amount of a resource to a randomly chosen subset of $(n-1) / 2$ other legislators, and each legislator's expected payoff from rejection is

$$
r=\frac{1}{n}\left((1-\delta)\left(\sum_{i=1}^{n} q_{i}\right)+\delta\right) .
$$

Also let $s_{i}$ denote the probability that, conditional on not being the proposer, legislator $i$ is offered a positive amount of the resource, e.g., $s_{i}=1 / 2$ if all proposers randomize uniformly over subsets of legislators. In such an equilibrium, if $i$ offers a positive amount of resource to another legislator, that amount will simply be $r$. Thus, the optimal payoff 
to a legislator $i$ when selected to propose will be

$$
1-r\left(\frac{n-1}{2}\right)
$$

reflecting the fact that $i$ offers $r$ to $(n-1) / 2$ others and keeps the rest of the resource. Legislator $i$ 's continuation value in such an equilibrium would be

$$
v_{i}=\left(1-\rho_{i}\right) s_{i} r+\rho_{i}\left(1-\frac{r(n-1)}{2}\right),
$$

reflecting the fact that, when someone else proposes, $i$ is offered the amount $r$ with probability $s_{i}$. Then $i$ 's payoff from rejecting a proposal is

$$
(1-\delta) q_{i}+\delta\left(1-\rho_{i}\right) s_{i} r+\delta \rho_{i}\left(1-\frac{r(n-1)}{2}\right) .
$$

Setting this equal to $r$ and solving for $s_{i}$, we have

$$
\begin{aligned}
s_{i} & =\frac{r-(1-\delta) q_{i}-\delta \rho_{i}\left(1-\frac{r(n-1)}{2}\right)}{\delta\left(1-\rho_{i}\right) r} \\
& =\frac{r-\delta \rho_{i}\left(1-\frac{r(n-1)}{2}\right)}{\delta\left(1-\rho_{i}\right) r}-q_{i} \frac{1-\delta}{\delta\left(1-\rho_{i}\right) r} .
\end{aligned}
$$

For the case $q_{i}=\bar{q} \in[0,1 / n]$ for all $i \in N$, so the legislators have equal consumption $\bar{q}$ of the resource in the status quo, and each $\rho_{i}=1 / n$, we then have $s_{i}=1 / 2$ and

$$
r=(1-\delta) \bar{q}+\frac{\delta}{n}
$$

Thus, a subset of $(n-1) / 2$ non-proposers is chosen at random with each member offered $r$, defined above, while the proposer keeps $1-r(n-1) / 2$, or equivalently,

$$
1-\frac{(1-\delta)(n-1) \bar{q}}{2}+\frac{\delta(n-1)}{2 n}
$$

Setting $\bar{q}=0$, as in Baron and Ferejohn (1989), non-proposers are offered $r=\delta / n$ and the proposer keeps $1-\delta(n-1) / 2 n$. Setting $\bar{q}=1 / n$, so the legislators split the resource evenly in the status quo, non-proposers are offered $1 / n$ and the proposer keeps $(n+1) / 2 n$. Thus, increasing the status quo $\bar{q}$ decreases the rent of the proposer. If the recognition probabilities are close to $1 / n$ and the status quo is close to $q=(\bar{q}, \ldots, \bar{q})$, then the probability that $i$ is offered a positive amount of the resource is given by (5), and the amount of the offer is given by (4). The probabilities in (5) correspond to stationary equilibria whenever $s_{i} \in[0,1]$ for each $i .{ }^{10}$ Assuming $\sum_{i=1}^{n} q_{i}=1$, so the status quo is

10 A caveat is that $s_{i}$ is the marginal probability on $i$ being offered a positive amount of resource, derived from a distribution on subsets of size $(n-1) / 2$. Given an arbitrary $\left(s_{1}, \ldots, s_{n}\right)$, there is the question of whether these marginal probabilities can be derived from such a distribution. A necessary condition is that $\sum s_{i}=n / 2$, a condition satisfied by (5) when each $\rho_{i}=1 / n$. The condition is sufficient when $n=3$, but we leave the question of the general case open. 
Pareto efficient, the conditions for this are as follows. When $q_{i} \geq 1 / n$, then $s_{i} \geq 0$ if and only if

$$
\rho_{i}<\frac{2}{n+1} \quad \text { and } \quad \frac{2\left(n q_{i}-1\right)}{2-\rho_{i}(n+1)} \leq \frac{\delta}{1-\delta},
$$

and $s_{i} \leq 1$ is not binding. When $q_{i} \leq 1 / n$, then $s_{i} \geq 0$ if and only if either $\rho_{i}<2 /$ $(n+1)$ or

$$
\rho_{i}>\frac{2}{n+1} \quad \text { and } \quad \frac{2\left(n q_{i}-1\right)}{2-\rho_{i}(n+1)} \geq \frac{\delta}{1-\delta},
$$

and $s_{i} \leq 1$ if and only if

$$
\frac{2\left(n q_{i}-1\right)}{n-1} \leq \frac{\delta \rho_{i}}{1-\delta}
$$

When $\rho_{i}=q_{i}=1 / n$, as mentioned above, $s_{i}=1 / 2 \in[0,1]$ and the above conditions hold for all $\delta$. An implication is that we do not have equilibria of the conjectured form in certain cases, e.g., when $q_{i}>1 / n$ and $\rho_{i}$ is too high, or when $q_{i}>1 / n, \rho_{i}$ is low, but $\delta$ is too low. In the latter cases, there is no way to bring $i$ 's expected payoff of rejection down to $r$, and, in equilibrium, $i$ will never be offered a positive amount of the resource.

In contrast to the preceding models, the next admits a stationary equilibrium in which delay occurs with positive probability. Note that the legislators in this model are risk neutral.

Model 6: An equilibrium with delay. Let $n=3, X=[0,1]$, each $\rho_{i}=1 / 3, q=0$, let $\mathcal{D}$ be majority rule, $u_{1}(x)=1-x$, and $u_{2}(x)=u_{3}(x)=x$. These utility functions are concave and strictly quasi-concave but linear. It can be checked that there exists a no-delay stationary equilibrium here, but we look for a stationary equilibrium with delay of the following form: $p_{1}=0, A_{1}=[0, x(\gamma)], p_{2}=p_{3}=1$, and $A_{2}=A_{3}=[x(\gamma), 1]$. Using the expression for $\gamma$ in the Appendix, we have

$$
\begin{aligned}
& \gamma(\{0\})=1-\delta+\delta\left(\frac{\rho_{1}(1-\delta)}{1-\delta \rho_{1}}\right) \\
& \gamma(\{1\})=\delta\left(\frac{\rho_{2}+\rho_{3}}{1-\delta \rho_{1}}\right) .
\end{aligned}
$$

Thus,

$$
x(\gamma)=\delta\left(\frac{\rho_{2}+\rho_{3}}{1-\delta \rho_{1}}\right) .
$$

By risk neutrality, the acceptance sets so-defined satisfy weak dominance, and proposal strategies clearly satisfy sequential rationality. But, because legislator 1's proposal is rejected and the other legislators propose a socially acceptable policy distinct from the 
status quo, this equilibrium exhibits delay: with probability $\rho_{1}>0$, the status quo $q=0$ will be obtained for a finite number of periods and be replaced by $x=1$. To see how the logic of the equilibrium in Model 3 breaks down, note that by risk neutrality, the utility from the expected outcome upon rejection, $x(\gamma)$, is exactly equal to the continuation value $v_{i}$ for each legislator $i$. Thus, when legislator 1 is selected to propose, the legislator has no way to pull the policy outcome toward zero while providing legislators 2 and 3 with utility at least equal to their continuation values. Equilibrium delay would not be possible given these three legislators if we let just one, say legislator 2, have a strictly concave utility function: then legislator 1 could offer 2 a policy slightly to the left of $x(\gamma)$, and this would pass. ${ }^{11}$

Finally, we turn to a model in which the legislators use a supermajority voting rule to choose from a two-dimensional policy space. Recall that in Models 1-3, when the status quo was in the core, it was the only possible bargaining outcome. In Model 4, when the status quo was at $(0,0)$, the core point, it was proposed by at least one legislator, but others could propose other policies. In contrast, in the next model the status quo is in the core, yet it is proposed by no legislator. Moreover, the status quo is not even in the social acceptance set.

Model 7: An equilibrium in which the status quo lies in the core but is not socially acceptable. Let $n=5$, let $X \subseteq \mathfrak{R}^{2}$ with status quo $q$ as in Figure 1 , and let each $\rho_{i}=1 / 5$. Define the supermajority voting rule $\mathcal{D}$ so that four or more votes are required for passage: $\mathcal{D}=\{C \subseteq N|| C \mid \geq 4\}$. Let the utility functions $u_{i}$ have ideal points and circular indifference curves as in Figure 1. Let legislator 1 mix over $x$ and $y$ with equal probabilities, and let each legislator $i=2,3,4,5$ propose $p_{i}$, as in Figure 1. We further specify utilities as follows. Defining

$$
v_{i}=\frac{u_{i}(x)+u_{i}(y)}{10}+\frac{1}{5} \sum_{j=2}^{5} u_{i}\left(p_{j}\right)
$$

for each legislator, we require

$$
\begin{aligned}
u_{1}\left(p_{3}\right) & =u_{1}\left(p_{4}\right)=(1-\delta) u_{1}(q)+\delta v_{1} \\
u_{2}(x) & =(1-\delta) u_{2}(q)+\delta v_{2} \\
u_{3}(y) & =u_{3}\left(p_{5}\right)=u_{3}\left(p_{4}\right)=(1-\delta) u_{3}(q)+\delta v_{3} \\
u_{4}(x) & =u_{4}\left(p_{2}\right)=u_{4}\left(p_{3}\right)=(1-\delta) u_{4}(q)+\delta v_{4} \\
u_{5}(y) & =(1-\delta) u_{5}(q)+\delta v_{5} .
\end{aligned}
$$

It is clear that this is consistent with our assumption that utilities be concave. Letting $A_{i}$ consist of policies $x$ such that $u_{i}(x) \geq(1-\delta) u_{i}(q)+\delta v_{i}$, weak dominance is satisfied,

11 But if we replicate these legislators one time, for a total of six, and if we let just legislator 2 have a strictly concave utility function, then we still have delay: a proposal slightly to the left of $x(\gamma)$ would garner only three votes and would fail. 


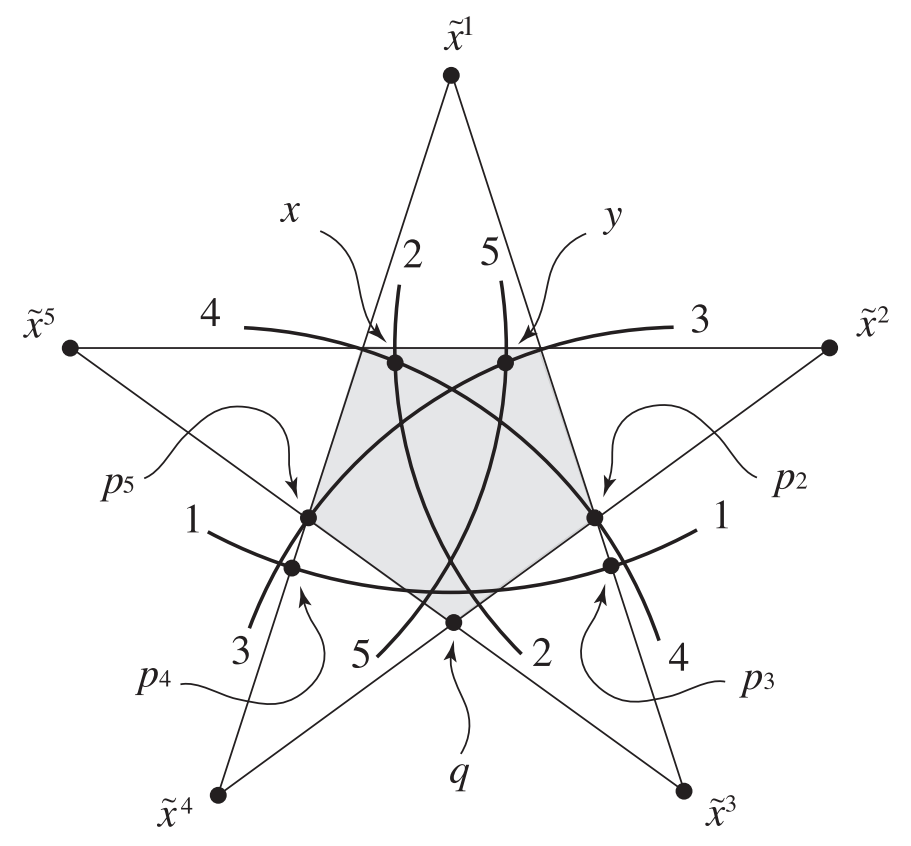

Figure 1. Legislator ideal points and proposals

and it is then clear from Figure 1 that sequential rationality is satisfied as well. Here, the core is the shaded polygon and contains the status quo, yet legislators 1,2 , and 5 would vote against the status quo if it were proposed.

\section{GENERAL RESULTS ON LEGISLATIVE BARGAINING}

Existence of Stationary Equilibria

In this subsection, we address two theoretical issues of fundamental importance for the applicability of the model described in the previous sections, namely, the existence and continuity properties of stationary equilibria. In each of the models of the previous section, a no-delay stationary equilibrium exists. Our first theorem establishes existence of no-delay stationary equilibria as a general result, despite the possibility that the status quo is favorable to some legislators.

Theorem 1 There exists a no-delay stationary equilibrium.

A further desirable property of equilibria is uniqueness. As Model 4 demonstrates, however, there may be multiple no-delay stationary equilibria, and in fact these need not even be payoff-equivalent. Cho and Duggan (2003) provide a unidimensional example 
with multiple stationary equilibria that are not payoff equivalent, so it is known that no general uniqueness result is available. ${ }^{12}$ The above existence proof uses mixed proposal strategies because, given stationary strategies for the other legislators, the set of proposals optimal for a legislator need not be convex. This possible non-convexity may arise because the set of socially acceptable proposals may itself be non-convex, as in Model 5. Thus, as in the theory of Nash equilibrium, mixing serves an important technical role in establishing existence of stationary equilibria.

In the unidimensional Models 1, 2, and 3, however, we found only pure strategy equilibria. The next theorem establishes this as a general result for unidimensional policy spaces. In fact, the result holds with no limit on the number of issues for a restricted class of voting rules. We say the voting rule $\mathcal{D}$ is oligarchic if there is a nonempty coalition $C \subseteq N$ of legislators that is decisive and is contained in every other decisive coalition, i.e., $\mathcal{D}=\left\{C^{\prime} \subseteq N \mid C \subseteq C^{\prime}\right\}$. This class of rules does not include majority rule, but it does contain the interesting case of unanimity rule. As another example, consider a majoritarian parliament where bargaining takes place among parties, which have weights in proportion to their size; if there is one party with more than half of the seats, then the voting rule is oligarchic.

Theorem 2 Assume either $d=1$ or the voting rule $\mathcal{D}$ is oligarchic. Every no-delay stationary equilibrium is in pure strategies.

This result simplifies the analysis of the bargaining model in a variety of environments, where we only need to work with pure proposal strategies, which are more tractable than mixed strategies. That Theorem 2 does not hold in multiple dimensions is demonstrated in Model 5, where we have found mixed strategy equilibria. Furthermore, Model 6 in Banks and Duggan (2005b) provides an example of mixed strategy equilibria in the multidimensional spatial setting, where legislators have quadratic utilities.

We next take up the issue of equilibrium continuity with respect to the parameters of our model, i.e., the recognition probabilities $\rho_{i}$, the status quo $q$, and the discount factor $\delta$. In addition, so that we may analyze the effect of varying legislator preferences, we parameterize the utility functions of the legislators by $\lambda$, which lies in a subset $\Lambda \subseteq \Re^{k}$ of finite-dimensional Euclidean space. That is, we expand the domain of the legislators' utility functions to $u_{i}: X \times \Lambda \rightarrow \Re$, and we assume that each $u_{i}$ is jointly continuous in $(x, \lambda)$, that each $u_{i}(\cdot, \lambda)$ is concave in $x$, and that LSWP is satisfied for all $\lambda \in \Lambda$. Thus, when we fix the parameter $\lambda$ at some value, the implied utility functions $u_{i}(\cdot, \lambda)$ correspond to an instance of our model, as described above. As an example of such a parameterization, it could be that $\lambda$ is a vector $\left(\lambda_{1}, \ldots, \lambda_{n}\right)$ with each $\lambda_{i}$ representing the ideal point of a quadratic utility function for legislator $i$, i.e., $u_{i}(x, \lambda)=-\left\|x-\lambda_{i}\right\|^{2}{ }^{23}$ For parameters $\rho=\left(\rho_{1}, \ldots, \rho_{n}\right), q, \delta$, and $\lambda$, let $E(\rho, q, \delta, \lambda)$ denote the set of profiles

12 Cho and Duggan (2003) prove that there is exactly one no-delay stationary equilibrium under the assumption of quadratic utilities. More generally, they prove that the social acceptance sets of no-delay stationary equilibria are nested, and that the minimal and maximal equilibria are unique.

13 More generally, $\lambda_{i}$ might be the matrix defining weighted Euclidean distance utilities (see Hinich and Munger 1997). 
of no-delay stationary equilibrium mixed proposal strategies. Our next result formalizes the idea that small variations in $(\rho, q, \delta, \lambda)$ cannot lead the set $E(\rho, q, \delta, \lambda)$ of no-delay stationary equilibria to expand discontinuously. ${ }^{14}$

Theorem 3 The correspondence $E$ of no-delay stationary equilibria is upper hemicontinuous in the parameters of the model.

This continuity of the no-delay stationary equilibrium proposal strategies is familiar from the Romer-Rosenthal model (Model 1), where the agenda setter's proposal $p_{n}=$ $\tilde{x}_{m}+\left|q-\tilde{x}_{m}\right|$ is clearly continuous in $q$. Moreover, because there is a unique no-delay stationary equilibrium when $\rho_{n}=1$, no-delay stationary equilibrium proposals will be close to this when $\rho_{n}$ is close to one or when utility functions are close to quadratic. In the Krehbiel model (Model 2), continuity of the median's proposal with respect to the status quo is also apparent. And, because there is a unique no-delay stationary equilibrium when $\rho_{m}=1$, no-delay stationary equilibrium proposals will be close to this when $\rho_{m}$ is close to one or when utilities are close to quadratic. Continuity is also demonstrated in Model 3, where $\Delta$ is a continuous function of the discount factor, the median voter's ideal point, and the status quo. Similarly, continuity is exhibited in Model 5, where the probability of being offered a positive amount of resource and the amount of the offer are continuous in the parameters of the model.

That Theorem 3 does not extend to all stationary equilibria, including those with delay, can be seen from Model 6 . There, we found a stationary equilibrium in which legislator 1 proposes $p_{1}=0$, which is rejected. Indeed, we could have specified any $p_{1} \in[0, x(\gamma))$, as all of these proposal would also be rejected. However, fixing the others' strategies, $p_{1}=x(\gamma)$ does not constitute a stationary equilibrium. The reason is that this proposal would be accepted, increasing the continuation values of legislators 2 and 3 discontinuously, and then the acceptance sets $A_{2}=A_{3}=x(\gamma)$ would no longer satisfy weak dominance. We conclude that the set of all stationary equilibrium proposal strategies is not even closed.

\section{Stability of the Status Quo}

We now turn to the issue of stability of the status quo in the stationary equilibria of our bargaining model and, in particular, the possibility of delay. We have already established the existence of equilibria without delay, but Theorem 1 leaves open the possibility that there are other equilibria in which some proposals are rejected. This is an especially interesting issue because delay is difficult to explain in models of complete information, and because it is an empirically observable aspect of the bargaining process. Recall that, of the special cases with strictly concave utilities, the only equilibria with delay were in Model 3, where the status quo was at the median and there was a static equilibrium in which every proposal was rejected. Our next theorem establishes this as a general

14 A correspondence $\Phi: X \rightrightarrows Y$ is upper hemicontinuous if given each point $x$ in the domain and each open set $G$ containing $\Phi(x)$, the set inclusion $\Phi(x) \subseteq G$ is maintained by arbitrarily small perturbations of $x$. See the Appendix for a formal definition for the equilibrium correspondence $E$. 
result: if legislators put any positive weight on the future, and if utility functions are strictly concave, then every stationary equilibrium is either no-delay or static. Thus, the outcome of bargaining in the first period will be the outcome in every period, whether it is the status quo or some other policy immediately agreed upon.

As phrased above, this result applies to the classical spatial model, but not to environments with a district-specific good, such as divide-the-dollar environments and local public good economies, where strict concavity is generally violated. In the BaronFerejohn model, however, we again found no equilibria with delay. We extend this observation to environments in which the sum of utilities is strictly concave and in which limited transfers are possible. We say the condition of limited transferability is satisfied if, for every policy $x \in X$ and every legislator $i \in N, u_{i}(x)>\min \left\{u_{i}(y) \mid y \in X\right\}$ implies there is a policy $z \in X$ such that, for every other legislator $j \neq i$, we have $u_{j}(z)>u_{j}(x)$. The idea is that we can take goods away from legislator $i$ and spread them across the remaining legislative districts, making those legislators better off. This is clearly possible in local public good economies, and the sum of utilities will also be strictly concave if every legislator's utility is strictly concave in his/her own district's consumption.

Theorem 4 Assume $\delta>0$, and assume that (i) there is a decisive coalition $C$ all the members of which have strictly concave utility functions, i.e., $u_{i}$ is strictly concave for all $i \in C$, or that (ii) the sum of utilities $\sum_{i \in N} u_{i}$ is strictly concave and limited transferability is satisfied. Every stationary equilibrium is either no-delay or static.

Equivalently, every stationary equilibrium with delay is static. An important implication of Model 6 is that the assumption of strict concavity in the first part of Theorem 4 cannot be dropped completely: in that model we found an equilibrium in which delay occurs with positive probability and yet the status quo is eventually replaced by another policy with probability one. This shows that an interesting form of delay can be obtained in our model, but that the role of risk neutrality was crucial in that example. Moreover, the element of strict concavity in the second part cannot be dropped: if one legislator's status quo level of pork is too high in Model 5, then that legislator's proposal will always be rejected. That a positive discount factor is needed for Theorem 4 can be seen by modifying Model 3 with $\tilde{x}_{m-1}<q<\tilde{x}_{m}$ and $\delta=0$. In the equilibrium found there, legislator $m-1$ proposes $q$, but having $m-1$ propose $\tilde{x}_{m-1}$, which is rejected is also a stationary equilibrium: because $\delta=0$, this change does not affect continuation values or acceptance sets, and legislator $m-1$ is indifferent between that proposal and $q$, the optimal choice from within the social acceptance set.

We now consider the possibility that in equilibrium all legislators propose the same policy, meaning that there is no ex ante uncertainty about the outcome of bargaining. In Models 3 and 4, we saw only two instances of such equilibria: in Model 3, with $q=\tilde{x}_{m}$, and in Model 4, with every legislator proposing $q=(0,0)$. In both cases the equilibrium was static. Our next theorem shows that this connection holds generally and that the conditions required for this coincidence of proposals are quite restrictive: unless all legislators have the same ideal point, an equilibrium in which all legislators propose the same policy must be static. 
Theorem 5 Assume that at least two legislators have distinct ideal points. If $\sigma$ is a no-delay stationary equilibrium in which every legislator proposes the same policy, i.e., there exists $x \in X$ such that $\pi_{i}(\{x\})=1$ for all $i \in N$, then $\sigma$ is static.

Thus, delay (assuming $\delta>0$ ) and coincidence of proposals (assuming at least two legislators have distinct ideal points) each imply a stationary equilibrium is static: the status quo obtains with probability one in every period. How restrictive is this conclusion? We saw static equilibria in Models 3 and 4, but only when the status quo was in the core. Our next result establishes this as a general necessary and sufficient condition for existence of a static equilibrium, under a very weak restriction on recognition probabilities.

Theorem 6 Assume that every decisive coalition has positive recognition probability, i.e., $\sum_{i \in C} \rho_{i}>0$ for all $C \in \mathcal{D}$. There exists a static stationary equilibrium if and only if the status quo is in the core, i.e., $q \in K$.

The possibility of delay or coincidence of proposals therefore hinges, under quite weak conditions, on the possibility that the status quo lies in the core. When all legislators have the same ideal point, contrary to the assumptions of Theorem 5 , it is easy to construct a stationary equilibrium exhibiting coincidence of proposals that is not static: every legislator simply proposes the ideal point and votes for it. When some decisive coalition has zero recognition probability, contrary to the assumptions of Theorem 6 , we may have a situation in which $\tilde{x}_{i}=q$ and $\rho_{i}=1$ for some legislator, in which case every stationary equilibrium is static, even if $q \notin K$. Except in those cases, however, Theorems 5 and 6 establish very general conditions under which delay and coincidence of proposals each imply that the status quo is in the core. In particular, they are possible only if the core is nonempty, a knife-edge condition for majority rule when there are multiple issue dimensions. The next corollary on the possibility of delay follows directly from the above results.

Corollary 1 Assume that $\delta>0$, that $\rho_{i}>0$ for every legislator $i \in N$, and that the status quo is not in the core, i.e., $q \notin K$. If each $u_{i}$ is strictly concave, then there are no stationary equilibria with delay.

The corollary is most relevant for higher dimensional issue spaces and voting rules such as majority rule, for then the core is almost always empty and $q \notin k$ almost always holds. Our results have an even starker consequence when transfers are possible, for then, for most voting rules of interest, the core is always empty. We say the voting rule $\mathcal{D}$ is non-collegial if there is no legislator in every decisive coalition, i.e., $\bigcap_{C \in \mathcal{D}} C=\emptyset$. This is clearly true for majority rule and any supermajority rule short of unanimity.

Corollary 2 Assume that $\delta>0$, that $\rho_{i}>0$ for every legislator $i \in N$, and that $\mathcal{D}$ is non-collegial. If the sum of utilities $\sum_{i \in N} u_{i}$ is strictly concave and limited transferability is satisfied, then there are no stationary equilibria with delay. 
Of course, similar corollaries hold for the possibility that all legislators propose the same policy. We conclude that, for a very wide class of models, it will almost always be the case that at least two legislators propose different policies with positive probability and that the first proposal will pass.

\section{Core Selection}

Theorem 6 yields a general condition under which there exists at least one stationary equilibrium that selects from the core with probability one: it is sufficient that the status quo lies in the core. In this subsection, we strengthen this result significantly by showing that, in many environments, if the status quo lies in the core, then it is the outcome of every stationary equilibrium. Thus, for the case of majority rule, bargaining equilibria always select from the set of medians, providing a gametheoretic foundation for the well-known median voter theorem. Our result applies to very general voting rules when the policy space is unidimensional and to the comparatively limited class of oligarchic voting rules when there are multiple issue dimensions. Note that by Theorem 2, all stationary equilibria are in pure strategies under these conditions.

Theorem 7 Assume that $\rho_{i}>0$ for every legislator $i \in N$. Also assume that $d=1$ and $\mathcal{D}$ is proper or that $\mathcal{D}$ is oligarchic. For every no-delay stationary equilibrium $\sigma$, the following implications hold.

$$
\text { (i) } A=\{q\} \quad \Leftrightarrow \quad \text { (ii) } q \in K \quad \Rightarrow \quad \text { (iii) } q \in A \text {. }
$$

Moreover, if $\delta>0$, then $($ iii $) \Rightarrow($ ii $)$.

The implication (iii) $\Rightarrow$ (ii) shows that, if legislators put any positive weight on the future and the status quo is not in the core $(q \notin K)$, then the status quo lies outside the social acceptance set $(q \notin A)$. Thus, no legislator proposes the status quo in any no-delay stationary equilibrium. If voting is by unanimity rule, so that the core is the set of Pareto optimal policies, then this simply means that no legislator would propose a Pareto-dominated status quo. Under majority rule, however, it means that no legislator will propose the status quo if it lies outside the interval of medians, which of course may be quite small. That $\delta>0$ is needed for the result can be seen from Model 3 by setting $\delta=0$ : then legislator 1 proposes $p_{1}=q \in A$. That the result does not hold generally in multiple dimensions can be seen from Model 6 in Banks and Duggan (2005b), where the core is empty, yet legislator 1 proposes the status quo, which is in the social acceptance set.

By implication $(i i) \Rightarrow(i)$, if the status quo is in the core, then it is the only socially acceptable policy, and so it is the only possible proposal in a no-delay equilibrium. Adding Theorems 4 and 6, it will therefore be the only possible outcome of any stationary equilibrium, with or without delay. Thus, if the policy space is unidimensional or the voting rule is oligarchic, and if the status quo is in the core, then the outcome of bargaining necessarily lies in the core. If voting is by unanimity rule, this means that a Pareto 
optimal status quo will necessarily be the unique policy outcome. For majority rule in one dimension, this generalizes the conclusion from Model 3, where $q=\tilde{x}_{m}$ implied that every legislator proposes $\tilde{x}_{m}$, the unique equilibrium outcome. That the result does not hold generally in more than one dimension can be seen from Model 4 with $\lambda=4$, where the status quo is in the core but there is a no-delay equilibrium in which the status quo is only proposed by one legislator. In fact, even the weaker implication (ii) $\Rightarrow$ (iii) does not hold generally: Model 7 shows that, if we omit both the assumption of a single policy dimension and the assumption that the voting rule is oligarchic, then the status quo may be in the core yet lie outside the social acceptance set.

This core selection result is related to the issue of core equivalence, addressed in Banks and Duggan (2000). There, we show that, assuming perfectly patient legislators $(\delta=1)$, the stationary equilibrium outcomes coincide with the core when either the policy space is unidimensional or the voting rule lies in a restricted class. That result differs from from Theorem 7 in several ways. First, the core equivalence result of Banks and Duggan (2000) gives conditions under which every core policy can be supported as an equilibrium outcome, whereas we are presently selecting just one core policy, namely, the status quo. Second, the restriction on voting rules used for the multidimensional case in that paper is somewhat weaker than requiring $\mathcal{D}$ be oligarchic. Third, that paper relies on perfect patience, whereas we presently allow any discount factor. In fact, because of the assumption of a bad status quo in that paper, core equivalence is impossible there when the discount factor is strictly less than one. Lastly, our core selection result applies only when the status quo is in the core, a condition that cannot hold in a model with a bad status quo.

An easy implication of our core selection result in Theorem 7, with the continuity result of Theorem 3, is the following comparative static on the status quo: as in Model 3, when the status quo is close to the core, the proposals of the legislators will also be close to the core in every no-delay stationary equilibrium. In other words, moderate status quos lead to moderate equilibrium policy outcomes. Given a set $Y \subseteq X$ and a policy $x$, let $d(Y, x)=\sup \{d(x, y) \mid y \in Y\}$ measure the distance from $Y$ to $x$, where $d(x, y)$ is the usual Euclidean distance. Thus, $d\left(A^{k}, q\right) \rightarrow 0$ in the next theorem implies that social acceptance sets collapse to a single core point.

Theorem 8 Assume that $\rho_{i}>0$ for every legislator $i \in N$. Also assume that $d=1$ and $\mathcal{D}$ is proper or that $\mathcal{D}$ is oligarchic. Let $q^{k} \rightarrow q \in K$, and let $\left\{\sigma^{k}\right\}$ be a corresponding sequence of no-delay stationary equilibria with social acceptance sets $\left\{A^{k}\right\}$. Then $d\left(A^{k}, q\right) \rightarrow 0$.

By Theorems 5 and 6 , we know that, unless the status quo is in the core, stationary equilibrium proposals will not be concentrated on any one core point. As a consequence, if $q \notin K$ and there is only one core point (as in Model 3), then some non-core policies are necessarily proposed. The next result shows, however, that in many environments there will always be at least one core policy proposed and passed with positive probability. This gives us a weaker core selection result: in every stationary equilibrium, a core policy will be realized with positive probability. 
Theorem 9 Assume that $d=1$ and $\mathcal{D}$ is proper or that $\mathcal{D}$ is oligarchic. In every no-delay stationary equilibrium $\sigma$, some legislator proposes a policy in the core, i.e., $p_{i} \in K$ for some $i \in N$.

We saw no-delay stationary equilibria with non-core proposals in Model 3 when the status quo was outside the core, i.e., $q \neq \tilde{x}_{m}$. We also saw, however, that these proposals converged to the core as the discount factor approached one. The next result establishes this result as a general theorem, yielding an asymptotic core selection result as the discount factor approaches one, regardless of the location of the status quo. The result applies to very general voting rules when the policy space is unidimensional, and it holds for a class containing the oligarchic rules when there are multiple issue dimensions. We say a voting rule is collegial if there is at least one legislator who belongs to every decisive coalition, i.e., $\bigcap_{C \in \mathcal{D}} C \neq \emptyset$. This class includes all oligarchic voting rules, including unanimity rule. Given sets $Y, Z \subseteq X$, we let $D(Y, Z)=\inf \{d(Y, z) \mid z \in Z\}$ measure the distance from $Y$ to $Z$. Thus, the convergence result stated next, that $D\left(A^{k}, K\right) \rightarrow 0$, means that socially acceptable policies must be arbitrarily close to core policies as the discount factor approaches one.

Theorem 10 Assume that $\rho_{i}>0$ for every legislator $i \in N$. Also assume that $d=1$ and $\mathcal{D}$ is proper or that $\mathcal{D}$ is collegial. Let $\delta^{k} \rightarrow 1$, and let $\left\{\sigma^{k}\right\}$ be a corresponding sequence of no-delay stationary equilibria with social acceptance sets $\left\{A^{k}\right\}$. Then $D\left(A^{k}, K\right) \rightarrow 0$.

In other words, legislative patience necessarily leads to moderate equilibrium policy outcomes. The implications of Theorem 10 are strongest when the core is a small set. If the voting rule $\mathcal{D}$ is majority rule with an odd number of legislators, for example, then the core consists of the unique median ideal point of the legislators, and in that case the stationary equilibrium outcomes of our bargaining model approach the prediction of the median voter theorem as legislators exhibit greater patience. Though we do not model the elections that produce legislative policy-makers, this result has an interesting implication for the design of electoral institutions: if we view legislator utility functions as representative of their districts, and if we view the discount factor of a legislator as corresponding to the probability of reelection, then systems that offer a sufficient incumbency advantage will lead to moderate policies.

\section{CONCLUSION}

This paper contributes a general model of legislative bargaining in which the status quo is an arbitrary point in a multidimensional policy space. In contrast to other bargaining models, the status quo is not assumed to be bad, and delay may be Pareto efficient. And in contrast to the social choice approach, where the existence of core policies in multiple dimensions is a significant problem, we prove existence of no-delay stationary equilibria for any number of issue dimensions. We have investigated the conditions under which delay is possible and found that, for the environments most interesting in policy-making 
applications, delay will almost never occur. Finally, we have given sufficient conditions for core selection, the event that stationary equilibrium outcomes always lie in the core, and we have provided results on asymptotic core selection. Importantly, we have found that moderate status quos lead to moderate policy outcomes, and legislative patience also leads to moderation. While we have considered mainly foundational issues here, we have proposed a general framework in which more substantive questions, about the nature of public goods provided or the coalitions that form to pass proposals, for example, can be taken up in special cases with more structure.

\section{APPENDIX: TECHNICAL MATTERS}

To define LSWP, we denote $i$ 's weak and strict upper contour sets at $x$, respectively, by

$$
\begin{aligned}
& R_{i}(x)=\left\{y \in X \mid u_{i}(y) \geq u_{i}(x)\right\} \\
& P_{i}(x)=\left\{y \in X \mid u_{i}(y)>u_{i}(x)\right\}
\end{aligned}
$$

and we let

$$
R_{C}(x)=\bigcap_{i \in C} R_{i}(x) \quad \text { and } \quad R(x)=\bigcup_{C \in \mathcal{D}} R_{C}(x),
$$

with similar conventions used for $P_{C}(x)$ and $P(x)$. That is, $R_{C}(x)$ consists of the policies that every member of $C$ weakly prefers to $x$, and $R(x)$ consists of the policies weakly preferred to $x$ by all members of some decisive coalition, with similar interpretations of $P_{C}(x)$ and $P(x)$. Given $Y \subseteq X$, let $\bar{Y}$ denote the closure of $Y$. Then limited shared weak preference is satisfied if, for all $C \subseteq N$ and all $x \in X$,

$$
\left|R_{C}(x)\right|>1 \text { implies } R_{C}(x) \subseteq \overline{P_{C}(x)} .
$$

That is, if $y$ (distinct from $x$ ) is weakly preferred to $x$ by all members of a coalition $C$, then it can be approximated by policies that all members of $C$ strictly prefer to $x$. We have given sufficient conditions for LSWP in the second Section. To see why strict quasi-concavity implies LSWP, for example, suppose all members of coalition $C$ weakly prefer some policy $y$ to $x \neq y$. Then every convex combination of $x$ and $y$ (with positive weight on both) is strictly preferred to $x$ by all members of $C$. Letting the weight on $y$ go to one, we approximate $y$ by policies in $P_{C}(x)$, as required. Note that, with concavity, LSWP implies strict quasi-concavity when $d=1$.

The characterization of the core in unidimensional environments is well-known (cf. Austen-Smith and Banks, 1999). Let $\underline{x}$ be the lowest ideal point, subject to the constraint that the legislators to the right are not decisive, i.e., $C_{1}=\left\{i \in N \mid \tilde{x}_{i}>\underline{x}\right\} \notin \mathcal{D}$, and let $\bar{x}$ be the greatest ideal point subject to the constraint that the legislators to the left are not decisive, i.e., $C_{2}=\left\{i \in N \mid \tilde{x}_{i}<\bar{x}\right\} \notin \mathcal{D}$. Then $K=[\underline{x}, \bar{x}]$, which is nonempty as long as $\mathcal{D}$ is proper. 
Given a stationary strategy profile $\sigma$, the probability measure $v$ is defined as follows: given measurable $Y \subseteq X$,

$$
v(Y)=\frac{\sum_{j \in N} \rho_{j}\left[\pi_{j}(Y \cap A)+I_{Y}(q)(1-\delta) \pi_{j}(X \backslash A)\right]}{1-\delta \sum_{j \in N} \rho_{j} \pi_{j}(X \backslash A)},
$$

where $I_{Y}(q)$ is the indicator function that takes value one if $q \in Y$ and zero otherwise. The continuation distribution, $\gamma$, is then defined as $\gamma(Y)=(1-\delta) \mu(Y)+\delta \nu(Y)$, where $\mu$ is the point mass on $q$.

Finally, in the following proofs, we refer to a subset $G \subseteq X$ as open if it is open in the relative topology, i.e., if there is an open subset $G^{\prime}$ of $\Re^{d}$ such that $G=X \cap G^{\prime}$.

Theorem 1 There exists a no-delay stationary equilibrium.

Proof: To prove existence, let $\pi$ denote a profile of mixed proposal strategies, and define

$$
v_{i}(\pi)=\sum_{j \in N} \rho_{j} \int u_{i}(z) \pi_{j}(d z)
$$

which would be $i$ 's continuation value if each $\pi_{j}$ put probability one on socially acceptable proposals. Note that, since $u_{i}$ is continuous, $v_{i}(\pi)$ is continuous in the weak topology on $[\mathcal{P}(X)]^{n}$. For all $i \in N$, define

$$
A_{i}(\pi)=\left\{x \in X \mid u_{i}(x) \geq(1-\delta) u_{i}(q)+\delta v_{i}(\pi)\right\},
$$

and, following our earlier conventions, let

$$
A_{C}(\pi)=\bigcap_{i \in C} A_{i}(\pi) \quad \text { and } \quad A(\pi)=\bigcup_{C \in \mathcal{D}} A_{C}(\pi) .
$$

Letting $\gamma$ denote the continuation distribution corresponding to the stationary strategy profile $\sigma=\left(\left(\pi_{1}, A_{1}(\pi)\right), \ldots,\left(\pi_{n}, A_{n}(\pi)\right)\right)$, concavity implies $x(\gamma)=\int z d \gamma \in A_{i}(\pi)$ for all $i \in N$. These sets are compact by compactness of $X$ and continuity of $u_{i}$, and they are convex by convexity of $X$ and concavity of $u_{i}$. For all $C \in \mathcal{D}, A_{C}(\pi)$ is also nonempty, compact, and convex. And $A(\pi)=\bigcup_{C \in \mathcal{D}} A_{C}(\pi)$ is nonempty and compact, but not necessarily convex. That $A(\pi)$ is continuous as a correspondence follows from the arguments in the proof of Theorem 7 in Banks and Duggan (2000). For all $i \in N$, define the optimal proposal correspondence,

$$
M_{i}(\pi)=\arg \max \left\{u_{i}(x) \mid x \in A(\pi)\right\},
$$

constraining the proposer to $A(\pi)$. By the Theorem of the Maximum (Aliprantis and Border 1994, Theorem 14.30), $M_{i}$ has nonempty and compact values and is upper hemicontinuous. However, it is not necessarily convex-valued, since $A(\pi)$ is not necessarily convex. Let $B_{i}(\pi)=\mathcal{P}\left(M_{i}(\pi)\right)$ denote the set of mixtures of constrained optimal 
proposals for $i$, which defines a nonempty-, compact-, convex-valued, upper hemicontinuous correspondence (Aliprantis and Border 1994, Theorem 14.14). Define the correspondence $B:[\mathcal{P}(X)]^{n} \rightrightarrows[\mathcal{P}(X)]^{n}$ by

$$
B(\pi)=B_{1}(\pi) \times \cdots \times B_{n}(\pi) .
$$

This correspondence is also nonempty-, compact-, convex-valued and upper hemicontinuous (Aliprantis and Border 1994, Theorem 14.14). Since $[\mathcal{P}(X)]^{n}$ is convex and is compact in the weak topology, Glicksberg's theorem (Aliprantis and Border 1994, Corollary 14.50) yields a fixed point of $B$, which we denote $\pi^{*}=\left(\pi_{1}^{*}, \ldots, \pi_{n}^{*}\right)$. We claim that $\sigma^{*}=\left(\left(\pi_{1}^{*}, A_{1}\left(\pi^{*}\right)\right), \ldots,\left(\pi_{n}^{*}, A_{n}\left(\pi^{*}\right)\right)\right)$ is a no-delay stationary equilibrium. By construction, this profile satisfies weak dominance and each $\pi_{i}^{*}$ puts probability one on $i$ 's utility-maximizing policies in $A\left(\pi^{*}\right)$. We have left to check that a proposer $i$ cannot obtain a higher expected payoff by proposing a policy outside $A\left(\pi^{*}\right)$. That payoff is $(1-\delta) u_{i}(q)+\delta v_{i}\left(\sigma^{*}\right)$. Letting $\gamma^{*}$ denote the continuation distribution corresponding to $\pi^{*}$, concavity implies that payoff is less than or equal to $u_{i}\left(x\left(\gamma^{*}\right)\right)$. Since $x\left(\gamma^{*}\right) \in A\left(\pi^{*}\right)$, we have sequential rationality, as required.

Theorem 2 Assume either $d=1$ or the voting rule $\mathcal{D}$ is oligarchic. Every no-delay stationary equilibrium is in pure strategies.

Proof: First, assume that $d=1$ and $\mathcal{D}$ is proper. Using the notation from the proof of Theorem 1 , we first note that $A(\pi)$ is convex for all $\pi$. To see this, let $\bar{x}=\max A(\pi)$ and $\underline{x}=\min A(\pi)$, which exist by compactness of $A(\pi)$, and let $\bar{C}$ and $\underline{C}$ satisfy $\bar{x} \in A_{\bar{C}}(\pi)$ and $\underline{x} \in A_{\underline{C}}(\pi)$. Letting $\gamma=\sum_{i \in N} \rho_{i} \pi_{i}$, concavity implies $x(\gamma) \in[\underline{x}, \bar{x}]=\operatorname{conv} A(\pi)$. By concavity, we have $x(\gamma) \in A_{\bar{C}}(\pi) \cap A_{\underline{C}}(\pi)$. Take any $y \in \operatorname{conv} A(\pi)$, and suppose without loss of generality that $y \in[\underline{x}, x(\gamma)]$. Since $A_{\underline{C}}(\pi)$ is convex, by concavity, we have $y \in A_{\underline{C}}(\pi) \subseteq A(\pi)$, as required. If $\sigma$ is a no-delay stationary equilibrium with mixed proposal profile, then it follows that $A(\pi)$ is convex. By LSWP, each $u_{i}$ has a unique ideal point in $X$, and then $d=1$ and concavity yield strict quasi-concavity. Thus, each $u_{i}$ has a unique maximizer in $A(\pi)$, and $\pi_{i}$ must put probability one on that policy.

Now assume $\mathcal{D}$ is oligarchic, with $C=\bigcap_{C^{\prime} \in \mathcal{D}} C^{\prime}$. Let $\sigma$ be any no-delay stationary equilibrium. Then $A(\pi)=A_{C}(\pi)$. Take any $i \in N$, and suppose that distinct policies $x^{\prime}$ and $x^{\prime \prime}$ solve $\max \left\{u_{i}(x) \mid x \in A(\pi)\right\}$. Letting $\gamma$ denote the continuation distribution corresponding to $\sigma$, note that concavity implies $x(\gamma) \in A(\pi)$ and, therefore, $u_{i}\left(x^{\prime}\right)=$ $u_{i}\left(x^{\prime \prime}\right) \geq u_{i}(x(\gamma)) \geq(1-\delta) u_{i}(q)+\delta v_{i}(\sigma)$. Thus, $x^{\prime}, x^{\prime \prime} \in A_{C \cup\{i\}}(\pi)$. Let $C^{\prime}$ consist of the members $j$ of $C \cup\{i\}$ such that $u_{j}\left(x^{\prime}\right)>u_{j}\left(x^{\prime \prime}\right)$, and let $G$ be an open set around $x^{\prime}$ such that, for all $j \in C^{\prime}$ and all $y \in G$, we have $u_{j}(y)>u_{j}\left(x^{\prime \prime}\right)$. Since $x^{\prime \prime} \in A_{j}(\pi)$ for all such $j$, this implies $u_{j}(y)>(1-\delta) u_{j}(q)+\delta v_{j}(\sigma)$. Let $C^{\prime \prime}=(C \cup\{i\}) \backslash C^{\prime}$ consist of the members $j$ of $C \cup\{i\}$ such that $u_{j}\left(x^{\prime \prime}\right) \geq u_{j}\left(x^{\prime}\right)$. Of course, $i \in C^{\prime \prime}$. By LSWP and $x^{\prime} \in A_{C^{\prime \prime}}(\pi)$, there exists $z \in X$ such that, for all $j \in C^{\prime \prime}$, we have $u_{j}(z)>u_{j}\left(x^{\prime}\right) \geq(1-\delta) u_{j}(q)+\delta v_{j}(\sigma)$. Choosing $\alpha \in(0,1)$ small enough that $z_{\alpha}=(1-\alpha) x^{\prime}+\alpha x^{\prime \prime} \in G$, concavity implies that $u_{j}\left(z_{\alpha}\right)>(1-\delta) u_{j}(q)+\delta v_{j}(\sigma)$ for 
all $j \in C$. Thus, $z_{\alpha} \in A(\pi)$, and, since $i \in C^{\prime \prime}$, we have $u_{i}\left(z_{\alpha}\right)>u_{i}\left(x^{\prime}\right)$, a contradiction. Therefore, each $u_{i}$ has a unique maximizer in $A(\pi)$, and $\pi_{i}$ must put probability one on that policy.

Letting $\Delta$ denote the unit simplex in $\Re^{n}$, define the correspondence $E: \Delta \times X \times$ $[0,1) \times \Lambda \rightrightarrows[\mathcal{P}(X)]^{n}$ such that $E(\rho, q, \delta, \lambda)$ consists of the profiles of no-delay stationary mixed proposal strategies for model parameters $(\rho, q, \delta, \lambda)$. We say that $E$ is upper hemicontinuous if, for every $(\rho, q, \delta, \lambda)$ and every open set $Y \subseteq[\mathcal{P}(X)]^{n}$ with $E(\rho, q, \delta, \lambda) \subseteq Y$, there exists an open set $Z \subseteq \Delta \times X \times[0,1) \times \Lambda$ with $(\rho, q, \delta, \lambda) \in Z$ such that, for all $\left(\rho^{\prime}, q^{\prime}, \delta^{\prime}, \lambda^{\prime}\right) \in Z$, we have $E\left(\rho^{\prime}, q^{\prime}, \delta^{\prime}, \lambda^{\prime}\right) \subseteq Y$.

Theorem 3 The correspondence $E$ of no-delay stationary equilibria is upper hemicontinuous in the parameters of the model.

Proof: Given parameters $\rho$ and $\lambda$, and given a profile $\pi$ of mixed proposal strategies, define

$$
v_{i}(\pi, \rho, \lambda)=\sum_{j \in N} \int u_{i}(z, \lambda) \pi_{j}(d z)
$$

for all $i \in N$. This would be $i$ 's continuation value if each $\pi_{j}$ put probability one on the social acceptance set. By continuity of $u_{i}$ and Billingsley's (1968) Theorem 5.5, $v_{i}$ is jointly continuous. Define

$$
A_{i}(\pi, \rho, q, \delta, \lambda)=\left\{x \in X \mid u_{i}(x) \geq(1-\delta) u_{i}(q, \lambda)+\delta v_{i}(\pi, \rho, \lambda)\right\}
$$

and write $A_{C}(\pi, \rho, q, \delta, \lambda)$ and $A(\pi, \rho, q, \delta, \lambda)$ using the usual conventions. Letting $\gamma=\sum_{j \in N} \rho_{j} \pi_{j}$, concavity implies $x(\gamma) \in A_{i}(\pi, \rho, q, \delta, \lambda)$ for all $i \in N$, which implies that the correspondence $A$ has nonempty values. Continuity of $A$ follows the arguments in the proof of Theorem 7 in Banks and Duggan (2000). By the Theorem of the Maximum, the correspondence $M_{i}$ of constrained optimal proposals, defined by

$$
M_{i}(\pi, \rho, q, \delta, \lambda)=\arg \max \left\{u_{i}(x) \mid x \in A(\pi, \rho, q, \delta, \lambda)\right\},
$$

has nonempty and compact values and is upper hemicontinuous. It follows that the correspondence $B_{i}$, defined by $B_{i}(\pi, \rho, q, \delta, \lambda)=\mathcal{P}\left(M_{i}(\pi, \rho, q, \delta, \lambda)\right)$ also possesses these properties. Since $B_{i}$ has closed values and regular range as well, it has closed graph (Aliprantis and Border 1994, Theorem 14.11). Now, let $\left(\rho^{k}, q^{k}, \delta^{k}, \lambda^{k}\right) \rightarrow$ $\left(\rho^{\circ}, q^{\circ}, \delta^{\circ}, \lambda^{\circ}\right)$, and take any sequence $\left\{\pi^{k}\right\}$ such that $\pi^{k} \in E\left(\rho^{k}, q^{k}, \delta^{k}, \lambda^{k}\right)$ for all $k$. Suppose $\pi^{k} \rightarrow \pi^{\circ}$. Since $\pi_{i}^{k} \in B_{i}\left(\rho^{k}, q^{k}, \delta^{k}, \lambda^{k}\right)$ for all $k$ and since $B_{i}$ has closed graph, we see that $\pi_{i}^{\circ} \in B_{i}\left(\rho^{\circ}, q^{\circ}, \delta^{\circ}, \lambda^{\circ}\right)$ for all $i \in N$. We claim that $\sigma^{\circ}$, with $\sigma_{i}^{\circ}=\left(\pi_{i}^{\circ}, A_{i}\left(\pi^{\circ}, \rho^{\circ}, q^{\circ}, \delta^{\circ}, \lambda^{\circ}\right)\right)$ for all $i \in N$, is a no-delay stationary equilibrium. Clearly, legislator acceptance sets satisfy weak dominance. If $\pi_{i}^{\circ}$ does not satisfy sequential rationality, then $i$ 's expected payoff from proposing a rejected policy exceeds the payoff from $\pi_{i}^{\circ}$, i.e.,

$$
\left(1-\delta^{\circ}\right) u_{i}\left(q^{\circ}, \lambda^{\circ}\right)+\delta^{\circ} v_{i}\left(\pi^{\circ}, \rho^{\circ}, \lambda^{\circ}\right)>\int u_{i}(z) \pi_{i}^{\circ}(d z) .
$$


Since these payoffs are jointly continuous, however, this strict inequality must hold for high enough $k$, contradicting $\pi^{k} \in E\left(\rho^{k}, q^{k}, \delta^{k}, \lambda^{k}\right)$. Therefore, $\pi^{\circ} \in E\left(\rho^{\circ}, q{ }^{\circ}, \delta^{\circ}\right.$, $\left.\lambda^{\circ}\right)$, and we conclude that $E$ has closed graph. Since it has compact Hausdorff range space as well, it is upper hemicontinuous (Aliprantis and Border 1994, Theorem 14.12).

Theorem 4 Assume $\delta>0$, and assume that (i) there is a decisive coalition $C$ all the members of which have strictly concave utility functions, i.e., $u_{i}$ is strictly concave for all $i \in C$, or that (ii) the sum of utilities $\sum_{i \in N} u_{i}$ is strictly concave and limited transferability is satisfied. Every stationary equilibrium is either no-delay or static.

Proof: Assume $\delta>0$. First, assume there exists $C \in \mathcal{D}$ such that, for all $i \in C$, $u_{i}$ is strictly concave. Since $\delta<1$, the status quo has positive mass according to the continuation distribution $\gamma$. If $\sigma$ is not static, then, since $\delta>0, \gamma$ is not concentrated on $q$, so strict concavity implies

$$
u_{i}(x(\gamma))>(1-\delta) u_{i}(q)+\delta v_{i}(\sigma)
$$

for all $i \in C$. By continuity, there is an open set $G$ around $x(\gamma)$ such that the inequality (6) holds for all $x \in G$. Since $C \in \mathcal{D}$, we then have $G \subseteq A$. Now consider any legislator $j \in N$, and let $\tilde{x}^{j}$ be $j$ 's unique utility-maximizing policy. We claim that there exists $z \in G$ such that $u_{j}(z)>(1-\delta) u_{j}(q)+\delta v_{j}(\sigma)$. This is clearly true if $\tilde{x}^{j}=x(\gamma)$. Otherwise, we have $u_{j}\left(\tilde{x}^{j}\right)>u_{j}(x(\gamma)) \geq(1-\delta) u_{j}(q)+\delta v_{j}(\sigma)$, and the claim follows from concavity by setting $\alpha \in(0,1)$ small enough that $z=(1-\alpha) x(\gamma)+\alpha \tilde{x}^{j} \in G$. Therefore, by sequential rationality, we have $\pi_{j}(A)=1$, so that $\sigma$ is no-delay.

Now assume that $\sum_{i \in N} u_{i}$ is strictly concave and that limited transferability is satisfied. Since $\delta<1$, the status quo has positive mass according to the continuation distribution $\gamma$. If $\sigma$ is not static, then, since $\delta>0, \gamma$ is not concentrated on $q$, so strict concavity implies

$$
\sum_{i \in N} u_{i}(x(\gamma))>\sum_{i \in N}\left[(1-\delta) u_{i}(q)+\delta v_{i}(\sigma)\right]
$$

By concavity of each $u_{i}$, it follows that there exists $i \in N$ such that $u_{i}(x(\gamma))>$ $(1-\delta) u_{i}(q)+\delta v_{i}(\sigma)$, and sequential rationality then implies that $\pi_{i}(A)=1$. Furthermore, $u_{i}(x(\gamma))>\min \left\{u_{i}(y) \mid y \in X\right\}$. By limited transferability, there exists $z \in X$ such that, for all $j \neq i, u_{j}(z)>u_{j}(x(\gamma))$. Choose $\alpha \in(0,1)$ small enough that $z_{\alpha}=(1-\alpha) x(\gamma)+\alpha z$ satisfies $u_{i}\left(z_{\alpha}\right)>(1-\delta) u_{i}(q)+\delta v_{i}(\sigma)$. By concavity, we have $u_{j}\left(z_{\alpha}\right)>u_{j}(x(\gamma)) \geq(1-\delta) u_{j}(q)+\delta v_{j}(\sigma)$ for all $j \neq i$, so $z_{\alpha} \in A$, and sequential rationality then implies $\pi_{j}(A)=1$ for all $j \neq i$. Thus, $\sigma$ is no-delay, as required.

Theorem 5 Assume that at least two legislators have distinct ideal points. If $\sigma$ is a no-delay stationary equilibrium in which every legislator proposes the same policy, i.e., there exists $x \in X$ such that $\pi_{i}(\{x\})=1$ for all $i \in N$, then $\sigma$ is static. 
Proof: Let $\sigma$ be a no-delay stationary equilibrium for which there exists $x \in X$ such that, for all $i \in N, \pi_{i}(\{x\})=1$. Thus, the continuation distribution places probability $1-\delta$ on $q$ and probability $\delta$ on $x$. If $\sigma$ is not static, then $x \neq q$, and it follows that $x \neq x(\gamma)$. Let $C$ consist of the legislators $i$ such that $u_{i}(x) \geq(1-\delta) u_{i}(q)+\delta v_{i}(\sigma)$, i.e., $x \in A_{i}$. Since $\sigma$ is no-delay, $C \in \mathcal{D}$. Let $C^{\prime}$ consist of the members $j$ of $C$ such that $u_{j}(x)>u_{j}(x(\gamma))$, and let $G$ be any open set around $x$ such that, for all $j \in C^{\prime}$ and all $y \in G$, we have $u_{j}(y)>u_{j}(x(\gamma))$. By concavity, it follows that, for all $j \in C^{\prime}$ and all $y \in G$, we also have $u_{j}(y)>(1-\delta) u_{j}(q)+\delta v_{j}(\sigma)$. Let $C^{\prime \prime}=C \backslash C^{\prime}$ consist of the members $j$ of $C$ such that $u_{j}(x(\gamma)) \geq u_{j}(x)$. By LSWP, there exists $z \in X$ such that, for all $j \in C^{\prime \prime}$, we have $u_{j}(z)>u_{j}(x)$. Then choosing $\alpha \in(0,1)$ small enough that $z_{\alpha}=(1-\alpha) x+\alpha z \in G$, concavity implies $u_{j}\left(z_{\alpha}\right)>u_{j}(x) \geq(1-\delta) u_{j}(q)+\delta v_{j}(\sigma)$ for all $j \in C^{\prime \prime}$. Therefore, $u_{j}\left(z_{\alpha}\right)>(1-\delta) u_{j}(q)+\delta v_{j}(\sigma)$ for all $j \in C$, and, since $C \in \mathcal{D}$, weak dominance implies $z_{\alpha} \in A$. Suppose $C^{\prime \prime} \neq \emptyset$, and take $i \in C^{\prime \prime}$. Then $u_{i}\left(z_{\alpha}\right)>u_{i}(x)$ and $z_{\alpha} \in A$ contradict sequential rationality. Thus, $C=C^{\prime}$, and we have $u_{i}(x)>(1-\delta) u_{i}(q)+\delta v_{i}(\sigma)$ for all $i \in C$. Since $C \in \mathcal{D}$, weak dominance implies that $x$ is in the interior of $A$. Now suppose that $u_{j}(y)>u_{j}(x)$ for some $j \in N$ and $y \in X$. Then, for small enough $\beta \in(0,1)$, we have $(1-\beta) x+\beta y \in A$, and, by concavity, $u_{j}((1-\beta) x+\beta y)>u_{j}(x)$, contradicting sequential rationality. It follows that $x$ is maximal for every legislator $i$, but this contradicts our assumption that at least two legislators have distinct ideal points. Therefore, $\sigma$ is static.

Theorem 6 Assume that every decisive coalition has positive recognition probability, i.e., $\sum_{i \in C} \rho_{i}>0$ for all $C \in \mathcal{D}$. There exists a static stationary equilibrium if and only if the status quo is in the core, i.e., $q \in K$.

Proof: Let $\sigma$ be a static stationary equilibrium, and suppose $q \notin K$, i.e., there exist $C \in \mathcal{D}$ and $y \in X$ such that $u_{i}(y)>u_{i}(q)=v_{i}(\sigma)$ for all $i \in C$. By weak dominance, $y \in A_{C} \subseteq A$. Then, for all $i \in C$, sequential rationality implies $\pi_{i}(A \backslash\{q\})=1$. But, because $\sum_{i \in C} \rho_{i}>0$, this contradicts the assumption that $\sigma$ is static. Now let $q \in K$, and define $\sigma$ by having each $i \in N$ propose $p_{i}=q$ and accept $A_{i}=R_{i}(q)$. Since $v_{i}(\sigma)=u_{i}(q)$ for all $i \in N$, this profile satisfies weak dominance. If sequential rationality is violated, then there exist $i \in N$ and $y \in A$ such that $u_{i}(y)>u_{i}(q)$. But $y \in A$ implies $y \in A_{C}$ for some $C \in \mathcal{D}$, i.e., $u_{i}(y) \geq u_{i}(q)$ for all $i \in C$. By LSWP, however, there exists $z \in X$ such that $u_{i}(z)>u_{i}(q)$ for all $i \in C$, contradicting $q \in K$. Therefore, sequential rationality is satisfied, and $\sigma$ is a stationary equilibrium.

Corollary 2 Assume that $\delta>0$, that $\rho_{i}>0$ for every legislator $i \in N$, and that $\mathcal{D}$ is non-collegial. If the sum of utilities $\sum_{i \in N} u_{i}$ is strictly concave and limited transferability is satisfied, then there are no stationary equilibria with delay.

Proof: From Theorems 4 and 6, it suffices to show that the core is empty. Take any $x \in X$. If $u_{i}(x)=\min \left\{u_{i}(y) \mid y \in X\right\}$ for all $i \in N$, then take any alternative $z \in X \backslash\{x\}$, so that $z \in R_{N}(x)$. By LSWP, there exists $z^{\prime} \in P_{N}(x)$, i.e., $u_{i}\left(z^{\prime}\right)>u_{i}(z)$ 
for all $i \in N$. Since $N \in \mathcal{D}$, this shows $x \notin K$. If $u_{i}(x)>\min \left\{u_{i}(y) \mid y \in X\right\}$ for some $i \in N$, then limited transferability yields a policy $z \in X$ such that, for all $j \neq i$, $u_{j}(z)>u_{j}(x)$. Since $\mathcal{D}$ is non-collegial, there exists $C \in \mathcal{D}$ such that $i \notin C$. Therefore, $u_{j}(z)>u_{j}(x)$ for all $j \in C$, and it follows that $x \notin K$. We conclude that $K=\emptyset$, as required.

Theorem 7 Assume that $\rho_{i}>0$ for every legislator $i \in N$. Also assume that $d=1$ and $\mathcal{D}$ is proper or that $\mathcal{D}$ is oligarchic. For every no-delay stationary equilibrium $\sigma$, the following implications hold.

$$
\text { (i) } A=\{q\} \quad \Leftrightarrow \quad \text { (ii) } q \in K \quad \Rightarrow \quad \text { (iii) } q \in A .
$$

Moreover, if $\delta>0$, then (iii) $\Rightarrow($ ii $)$.

Proof: Let $\sigma$ be a no-delay stationary equilibrium. Note that $(i) \Rightarrow(i i)$ holds generally. Indeed, if $A=\{q\}$, then $\sigma$ is static. That $q \in K$ then follows from Theorem 6 . The remainder of the proof is divided into two parts corresponding to the two sets of assumptions in the theorem.

First, assume $d=1$ and $\mathcal{D}$ is proper. We begin by showing (ii) $\Rightarrow$ (iii). Suppose $q \in K$, denote the ideal points of the legislators by $\tilde{x}_{i}$, and let $\tilde{x}_{1} \leq \tilde{x}_{2} \leq \cdots \leq \tilde{x}_{n}$. Then there exist $m_{1}, m_{2} \in N$ such that $K=\left[\tilde{x}_{m_{1}}, \tilde{x}_{m_{2}}\right], C_{1}=\left\{i \in N \mid i>m_{1}\right\} \notin \mathcal{D}$, and $C_{2}=\left\{i \in N \mid i<m_{2}\right\} \notin \mathcal{D}$. Let $A=[\underline{x}, \bar{x}]$. If $\underline{x}=\bar{x}$, then all legislators propose the same policy and, as long as some legislators have distinct ideal points, Theorem 5 implies $q \in A$; if all legislators have the same ideal point, then $q \in K$ implies that ideal point is $q$, and again $q \in A$. So consider the case $\underline{x}<\bar{x}$, and suppose $q \notin A$. In particular, suppose $q<\underline{x}$ without loss of generality. Take any $i \leq m_{1}$, and note that $\bar{x}$ minimizes $u_{i}$ over $A$. Since $\sigma$ is no-delay, (2) implies that $v_{i}(\sigma) \geq u_{i}(\bar{x})$. Furthermore, $q \in K$ implies $u_{i}(q)>u_{i}(\bar{x})$, so we have $u_{i}(\bar{x})<(1-\delta) u_{i}(q)+\delta v_{i}(\sigma)$, and then $\bar{x} \notin A_{i}$ by weak dominance. Therefore, the legislators who accept $\bar{x}$ are contained in $C_{1}$, and it follows that $\bar{x} \notin A$, a contradiction. Therefore, $q \in A$. We now suppose $\delta>0$ and show (iii) $\Rightarrow(i)$. From the above, this will imply (iii) $\Rightarrow(i i)$, as in the theorem. Suppose $q \in A$. Let $\underline{x}=\min A$ and $\bar{x}=\max A$, and let $\underline{C}, \bar{C} \in \mathcal{D}$ satisfy $\underline{x} \in A_{\underline{C}}$ and $\bar{x} \in A_{\bar{C}}$. Since $\mathcal{D}$ is proper, there exists $i \in \underline{C} \cap \bar{C}$. Thus,

$$
\min \left\{u_{i}(\underline{x}), u_{i}(\bar{x})\right\} \geq(1-\delta) u_{i}(q)+\delta v_{i}(\sigma)=\int u_{i}(z) \gamma(d z)
$$

Since $\sigma$ is no-delay, $\gamma(A \cup\{q\})=1$. Since $q \in A$, we have $\gamma(A)=1$. Since $u_{i}$ is concave, it attains its minimum over $A$ at one of $\underline{x}$ or $\bar{x}$, i.e.,

$$
\min \left\{u_{i}(x) \mid x \in A\right\}=\min \left\{u_{i}(\underline{x}), u_{i}(\bar{x})\right\} .
$$

Thus, $\gamma$ must put probability one on $i$ 's utility-minimizing socially acceptable proposals, i.e.,

$$
\gamma\left(\arg \min \left\{u_{i}(x) \mid x \in A\right\}\right)=1 .
$$


As in the proof of Theorem $2, A$ is convex, so $(1 / 2) \underline{x}+(1 / 2) \bar{x} \in A$. Then sequential rationality implies

$$
u_{i}\left(p_{i}\right) \geq u_{i}((1 / 2) \underline{x}+(1 / 2) \bar{x})>\min \left\{u_{i}(\underline{x}), u_{i}(\bar{x})\right\}=\min \left\{u_{i}(x) \mid x \in A\right\},
$$

where the strict inequality above follows from strict quasi-concavity. But this, $\delta>0$, and $\rho_{i}>0$, contradict (7). Therefore, $\underline{x}=\bar{x}$ and $A=\{q\}$. Finally, we show $(i i) \Rightarrow(i)$. Suppose $q \in K$. From the above, $A=\{q\}$ follows if $\delta>0$, so suppose $\delta=0$. Again let $\tilde{x}_{1} \leq \tilde{x}_{2} \leq \cdots \leq \tilde{x}_{n}$ and $K=\left[\tilde{x}_{m_{1}}, \tilde{x}_{m_{2}}\right]$, and consider any $x<q$. By weak dominance, $A_{i}=R_{i}(q)$, and, by concavity, the legislators who accept $x$ therefore satisfy $\tilde{x}_{i}<q$. Thus, $i$ accepts $x$ only if $\tilde{x}_{i}<\tilde{x}_{m_{2}}$, and this coalition cannot be decisive. We conclude that $x \notin A$, and a similar argument holds for $x>q$, as required.

Now assume that $\mathcal{D}$ is oligarchic, and let $C=\bigcap_{C^{\prime} \in \mathcal{D}} C^{\prime}$. If $u_{i}(q) \geq v_{i}(\sigma)$ for some $i \in C$, then we claim that $q=p_{j}$ for all $j \in N$. Since $\sigma$ is no-delay and $\rho_{j}>0$ for all $j \in N$, we have $p_{j} \in A=A_{C}$ for all $j$. Since $i \in C$, it follows that $u_{i}\left(p_{j}\right) \geq(1-\delta) u_{i}(q)+\delta v_{i}(\sigma)$ for all $j \in N$. Then

$$
v_{i}(\sigma)=\sum_{j \in N} \rho_{j} u_{i}\left(p_{j}\right) \geq(1-\delta) u_{i}(q)+\delta v_{i}(\sigma)
$$

implies that $v_{i}(\sigma) \geq u_{i}(q)$, so we have $v_{i}(\sigma)=u_{i}(q)$. Furthermore, $p_{j} \in A_{i}$ becomes $u_{i}\left(p_{j}\right) \geq u_{i}(q)$, and then $\rho_{j}>0$ for all $j \in N$ and

$$
v_{i}(\sigma)=\sum_{j \in N} \rho_{j} u_{i}\left(p_{j}\right) \geq u_{i}(q)=v_{i}(\sigma)
$$

imply that $u_{i}\left(p_{j}\right)=u_{i}(q)$ for all $j \in N$. If it is not the case that all legislators propose $q$, then there exists $j \in N$ such that $p_{j} \neq x(\gamma)$. Let $C^{\prime}$ consist of the members $h$ of $C$ such that $u_{h}\left(p_{j}\right)>u_{h}(x(\gamma))$, and let $G$ be an open set around $p_{j}$ such that, for all $h \in C^{\prime}$ and all $y \in G$, we have $u_{h}(y)>u_{h}(x(\gamma))$. By concavity, for all $h \in C^{\prime}$ and all $y \in G$, we also have $u_{h}(y)>(1-\delta) u_{h}(q)+\delta v_{h}(\sigma)$. Let $C^{\prime \prime}=C \backslash C^{\prime}$ consist of the members $h$ of $C$ such that $u_{h}(x(\gamma)) \geq u_{h}\left(p_{j}\right)$. Of course, $i \in C$. By LSWP, there exists $z \in X$ such that, for all $h \in C^{\prime \prime}$, we have $u_{h}(z)>u_{h}\left(p_{j}\right)$. Choosing $\alpha \in(0,1)$ small enough that $z_{\alpha}=(1-\alpha) p_{j}+\alpha z \in G$, concavity implies that $u_{h}\left(z_{\alpha}\right)>u_{h}\left(p_{j}\right) \geq$ $(1-\delta) u_{h}(q)+\delta v_{h}(\sigma)$ for all $h \in C$. Thus, $u_{h}\left(z_{\alpha}\right) \geq(1-\delta) u_{h}(q)+\delta v_{h}(\sigma)$ for all $h \in C$. Since $C \in \mathcal{D}$, this implies $z_{\alpha} \in A$. Then sequential rationality implies that

$$
u_{i}\left(p_{i}\right) \geq u_{i}\left(z_{\alpha}\right)>u_{i}\left(p_{j}\right)=u_{i}(q),
$$

where the strict inequality follows from $i \in C$, contradicting $u_{i}\left(p_{i}\right)=u_{i}(q)$. Therefore, $u_{i}(q) \geq v_{i}(\sigma)$ implies $p_{j}=q$ for all $j \in N$.

We now show (ii) $\Rightarrow($ iii). Suppose $q \in K$ but $q \notin A$. Then there exists $j \in C$ such that $u_{j}(q)<(1-\delta) u_{j}(q)+\delta v_{j}(\sigma)$, so $\delta>0$ and, by concavity, $u_{j}(q)<u_{j}(x(\gamma))$. Since $q \in K$, there exists $i \in C$ such that $u_{i}(q) \geq u_{i}(x(\gamma)) \geq(1-\delta) u_{i}(q)+\delta v_{i}(\sigma)$, and, since $\delta>0$, we have $u_{i}(q) \geq v_{i}(\sigma)$. Thus, we have $p_{j}=q$ for all $j \in N$, but 
then $x(\gamma)=q$, contradicting $u_{j}(q)<u_{j}(x(\gamma))$. Therefore, $q \in A$. We next show (iii) $\Rightarrow(i)$, assuming $\delta>0$. From the above, this will imply (iii) $\Rightarrow($ ii $)$, as in the theorem. If $q \in A$, then, for all $i \in C$, we have $u_{i}(q) \geq(1-\delta) u_{i}(q)+\delta v_{i}(\sigma)$. Since $\delta>0$, this implies $u_{i}(q) \geq v_{i}(\sigma)$, so again $p_{j}=q$ for all $j \in N$. Suppose there exists $x \in A$ with $x \neq q$. Then $u_{i}(x) \geq(1-\delta) u_{i}(q)+\delta v_{i}(\sigma)=u_{i}(q)$ for all $i \in C$. By LSWP, there exists $z \in X$ such that $u_{i}(z)>u_{i}(q)$ for all $i \in C$, and therefore $z \in A$. Then sequential rationality implies that, for all $i \in C$, we have $u_{i}\left(p_{i}\right) \geq u_{i}(z)>u_{i}(q)=u_{i}\left(p_{i}\right)$, a contradiction. Therefore, $A=\{q\}$. Finally, we show (ii) $\Rightarrow(i)$. Suppose $q \in K$. From the above, $A=\{q\}$ follows if $\delta>0$, so let $\delta=0$. Suppose there exists $x \in A$ with $x \neq q$, so $u_{i}(x) \geq u_{i}(q)$ for all $i \in C$. But then, by LSWP, there exists $z \in X$ such that $u_{i}(z)>u_{i}(q)$ for all $i \in C$, contradicting $q \in K$. Therefore, $A=\{q\}$, as required.

Theorem 8 Assume that $d=1$ and $\mathcal{D}$ is proper or that $\mathcal{D}$ is oligarchic. In every no-delay stationary equilibrium $\sigma$, some legislator proposes a policy in the core, i.e., $p_{i} \in K$ for some $i \in N$.

Proof: First, assume $d=1$ and $\mathcal{D}$ is proper. Let $A=[\underline{x}, \bar{x}]$. Denote the ideal points of the legislators by $\tilde{x}_{i}$, and let $\tilde{x}_{1} \leq \tilde{x}_{2} \leq \cdots \leq \tilde{x}_{n}$. Since $d=1$ and $\mathcal{D}$ is proper, there exist $m_{1}, m_{2} \in N$ such that $K=\left[\tilde{x}_{m_{1}}, \tilde{x}_{m_{2}}\right], C_{1}^{+}=\left\{i \in N \mid \tilde{x}_{i} \geq \tilde{x}_{m_{1}}\right\} \in \mathcal{D}$, and $C_{2}^{+}=\left\{i \in N \mid \tilde{x}_{i} \leq \tilde{x}_{m_{2}}\right\} \in \mathcal{D}$. If $\tilde{x}_{m_{2}}<x(\gamma)$, then, for all $i \in C_{2}$, we have

$$
u_{i}\left(\tilde{x}_{m_{2}}\right) \geq u_{i}(x(\gamma)) \geq(1-\delta) u_{i}(q)+\delta v_{i}(\sigma),
$$

i.e., $\tilde{x}_{m_{2}} \in A_{C_{2}}$. Since $C_{2} \in \mathcal{D}$, we have $\tilde{x}_{m_{2}} \in A$, and, by sequential rationality, $p_{m_{2}}=\tilde{x}_{m_{2}}$. Similarly, if $x(\gamma)<\tilde{x}_{m_{1}}$, then $\tilde{x}_{m_{1}} \in A$ and $p_{m_{1}}=\tilde{x}_{m_{1}}$. If $\tilde{x}_{m_{1}} \leq x(\gamma) \leq \tilde{x}_{m_{2}}$, then, since $x(\gamma) \in A$, we have $A \cap K \neq \emptyset$. Then, by sequential rationality, $p_{m_{1}}=\tilde{x}_{m_{1}}$ if it is in $A$ or $p_{m_{1}}=\min A \in K$ otherwise.

Now assume $\mathcal{D}$ is oligarchic, with $C=\bigcap_{C^{\prime} \in \mathcal{D}} C^{\prime}$, and consider $i \in C$ and any proposal $p_{i} \in A$ for legislator $i$. If $p_{i} \notin K$, then there exists $x \in X$ such that, for all $j \in C, u_{j}(x)>u_{j}\left(p_{i}\right)$. Since $p_{i} \in A$, there exists $C^{\prime} \in \mathcal{D}$ such that, for all $j \in C^{\prime}, u_{j}\left(p_{i}\right) \geq(1-\delta) u_{j}(q)+\delta u_{j}(p)$. Since $C \subseteq C^{\prime}$, we have $u_{j}(x)>$ $(1-\delta) u_{j}(q)+\delta u_{j}(p)$ for all $j \in C$, which implies $x \in A$. But then $u_{i}(x)>u_{i}\left(p_{i}\right)$ violates sequential rationality. Therefore, we must have $p_{i} \in K$ in every no-delay equilibrium.

Theorem 9 Assume that $\rho_{i}>0$ for every legislator $i \in N$. Also assume that $d=1$ and $\mathcal{D}$ is proper or that $\mathcal{D}$ is collegial. Let $\delta^{k} \rightarrow 1$, and let $\left\{\sigma^{k}\right\}$ be a corresponding sequence of no-delay stationary equilibria with social acceptance sets $\left\{A^{k}\right\}$. Then $D\left(A^{k}, K\right) \rightarrow 0$.

Proof: In case $\mathcal{D}$ is collegial, specify $i \in \bigcap_{C \in \mathcal{D}} C$ arbitrarily, so that $A^{k} \subseteq A_{i}^{k}$ for all $k$. Since each $\sigma^{k}$ is no-delay, this implies that

$$
\pi_{j}^{k}\left(\left\{x \in X \mid u_{i}(x) \geq\left(1-\delta^{k}\right) u_{i}(q)+\delta^{k} v_{i}\left(\sigma^{k}\right)\right\}\right)=1
$$


for every legislator $j \in N$ and for all $k$. In case $d=1$ and $\mathcal{D}$ is proper, let $y^{k}=\min A^{k}$ and $z^{k}=\max A^{k}$ for all $k$, and let $\underline{C}^{k}, \bar{C}^{k} \in \mathcal{D}$ satisfy $y^{k} \in A_{\underline{C}^{k}}$ and $z^{k} \in A_{\bar{C}^{k}}$. Since $\mathcal{D}$ is proper, there exists $i^{k} \in \underline{C}^{k} \cap \bar{C}^{k}$. Going to a subsequence if necessary, we may assume that $i^{k}=i$ for all $k$. Thus, we have

$$
\min \left\{u_{i}(x) \mid x \in A^{k}\right\}=\min \left\{u_{i}\left(y^{k}\right), u_{i}\left(z^{k}\right)\right\} \geq\left(1-\delta^{k}\right) u_{i}(q)+\delta^{k} v_{i}\left(\sigma^{k}\right)
$$

for all $k$, where the first equality uses convexity of $A^{k}$, established in the proof of Theorem 2, and concavity of $u_{i}$. Since $\pi_{j}^{k}\left(A^{k}\right)=1$ for all $j$ and $k$, we again have (8). Thus, there exists a subsequence of $\left\{\sigma^{k}\right\}$ for which (8) holds for all $j$ and all $k$. In the next three paragraphs, we consider such a subsequence and deduce further properties of it.

Define $w_{i}^{k}(j)$ to be the expected payoff to legislator $i$ when $j$ is selected to propose in the equilibrium $\sigma^{k}$, i.e.,

$$
w_{i}^{k}(j)=\int u_{i}(x) \pi_{j}^{k}(d x),
$$

and note that $v_{i}\left(\sigma^{k}\right)=\sum_{j \in N} \rho_{j} w_{i}^{k}(j)$. Letting

$$
\ell^{k} \in \arg \min \left\{w_{i}^{k}(j) \mid j \in N\right\} \quad \text { and } \quad h^{k} \in \arg \max \left\{w_{i}^{k}(j) \mid j \in N\right\},
$$

Equations 8 and 9 imply

$$
\begin{aligned}
w_{i}^{k}\left(\ell^{k}\right) & \geq\left(1-\delta^{k}\right) u_{i}(q)+\delta^{k} \rho_{h^{k}} w_{i}^{k}\left(h^{k}\right)+\delta^{k} \sum_{j \neq h^{k}} \rho_{j} w_{i}^{k}(j) \\
& \geq\left(1-\delta^{k}\right) u_{i}(q)+\delta^{k} \rho_{h^{k}} w_{i}^{k}\left(h^{k}\right)+\delta^{k}\left(1-\rho_{h^{k}}\right) w_{i}^{k}\left(\ell^{k}\right),
\end{aligned}
$$

which implies that

$$
\min _{j \in N} w_{i}^{k}(j) \geq \max _{j \in N} w_{i}^{k}(j)+\frac{1-\delta^{k}}{\delta^{k} \rho_{h^{k}}}\left(u_{i}(q)-w_{i}^{k}\left(\ell^{k}\right)\right)
$$

for all $k$. Since $\mathcal{P}(X)$ is compact in the weak topology, the sequence $\left\{\left(\pi_{1}^{k}, \ldots, \pi_{n}^{k}\right)\right\}$ has a convergent subsequence (also indexed by $k$ ) with limit, say, $\left(\pi_{1}, \ldots, \pi_{n}\right)$. For all $j \in N$, define $w_{i}(j)=\int u_{i} d \pi_{j}$. Since $u_{i}$ is continuous, weak convergence implies $w_{i}^{k}(j) \rightarrow w_{i}(j)$, and then (10) implies that $w_{i}(j)$ is independent of $j$, i.e., there exists $w_{i}$ such that $w_{i}(j)=w_{i}$ for all $j \in N$. In general, for any legislator $j$, weak convergence implies that the sequence $\left\{v_{j}\left(\sigma^{k}\right)\right\}$ of continuation values converges to

$$
v_{j}=\lim v_{j}\left(\sigma^{k}\right)=\sum_{h \in N} \rho_{h} \int u_{j} d \pi_{h},
$$

and for legislator $i$ we have

$$
v_{i}=\lim v_{i}\left(\sigma^{k}\right)=\lim \sum_{j \in N} \rho_{j} w_{i}^{k}(j)=w_{i}
$$

as well. Letting $\gamma^{k}$ be the continuation distribution corresponding to $\sigma^{k}$, concavity implies $x\left(\gamma^{k}\right) \in A_{j}^{k}$ for all $j$ and $k$, or in other words $u_{j}\left(x\left(\gamma^{k}\right)\right) \geq\left(1-\delta^{k}\right) u_{j}(q)+$ 
$\delta^{k} v_{j}\left(\sigma^{k}\right)$ for all $j$ and $k$. Letting $\gamma$ denote the limit of continuation distributions, which is just $\sum_{j \in N} \rho_{j} \pi_{j}$, weak convergence implies $x\left(\gamma^{k}\right) \rightarrow x(\gamma)$, the mean of the probability measure $\gamma$, and then, by continuity, we have $u_{j}(x(\gamma)) \geq v_{j}$ for all $j \in N$.

We claim that, for all $j \in N, \pi_{j}$ is the point mass on $x(\gamma)$. If not, then there exists an open set $G$ around $x(\gamma)$, a legislator $j$, and a subsequence of $\left\{\left(\pi_{1}^{k}, \ldots, \pi_{n}^{k}\right)\right\}$ (still indexed by $k$ ) such that, for all $k$, we have $\pi_{j}^{k}(X \backslash G)>0$. Letting $S^{k}$ denote the support of $\pi_{j}^{k}$, this is equivalent to $S^{k} \backslash G \neq \emptyset$ for all $k$. Thus, for each $k$, there exists $x^{k} \in S^{k} \backslash G$, and going to a subsequence (still indexed by $k$ ) if needed, we assume that $x^{k} \rightarrow x$. For all $k$, since $\sigma^{k}$ is no-delay, there exists $C^{k} \in \mathcal{D}$ such that $x^{k} \in A_{C}^{k}$. Note that $i \in C^{k}$ by (8). Again going to a subsequence (still indexed by $k$ ) if needed, we may assume that $C^{k}=C$ for all $k$, and of course $i \in C$. Thus, $u_{h}\left(x^{k}\right) \geq\left(1-\delta^{k}\right) u_{h}(q)+\delta^{k} v_{h}\left(\sigma^{k}\right)$ for all $h \in C$. An implication of continuity and (11) is then that $u_{h}(x) \geq v_{h}$ for all $h \in C$. Let $C^{\prime}$ consist of the members $h$ of $C$ such that $u_{h}(x)>u_{h}(x(\gamma))$, and let $G^{\prime}$ be any open set around $x$ such that, for all $h \in C^{\prime}$ and all $y \in G^{\prime}$, we have $u_{h}(y)>u_{h}(x(\gamma))$. It follows that, for all $h \in C^{\prime}$ and all $y \in G^{\prime}$, we also have $u_{h}(y)>v_{h}$. Let $C^{\prime \prime}=C \backslash C^{\prime}$ consist of the members $h$ of $C$ such that $u_{h}(x(\gamma)) \geq u_{h}(x)$. By LSWP, there exists $z \in X$ such that, for all $h \in C^{\prime \prime}$, we have $u_{h}(z)>u_{h}(x)$. Then choosing $\alpha \in(0,1)$ small enough that $z_{\alpha}=(1-\alpha) x+\alpha z \in G^{\prime}$, concavity implies $u_{h}\left(z_{\alpha}\right)>u_{h}(x) \geq v_{h}$ for all $h \in C^{\prime \prime}$. Therefore, $\epsilon=\min _{h \in C}\left(u_{h}\left(z_{\alpha}\right)-v_{h}\right)>0$, and by (11) we have

$$
u_{h}\left(z_{\alpha}\right) \geq\left(1-\delta^{k}\right) u_{h}(q)+\delta^{k} v_{h}\left(\sigma^{k}\right)+\frac{\epsilon}{2}
$$

for all $h \in C$ and for high enough $k$. Since $C \in \mathcal{D}$, it follows from weak dominance that $z_{\alpha} \in A^{k}$, and then, since $i \in C$, sequential rationality then implies that

$$
w_{i}^{k}(i) \geq\left(1-\delta^{k}\right) u_{h}(q)+\delta^{k} v_{i}\left(\sigma^{k}\right)+\frac{\epsilon}{2} .
$$

But this inequality, with (12), yields

$$
w_{i}=\lim w_{i}^{k}(i) \geq \lim v_{i}^{k}+\frac{\epsilon}{2}=v_{i}+\frac{\epsilon}{2}>w_{i},
$$

a contradiction. Thus, each $\pi_{j}^{k}$ indeed converges to the point mass on $x(\gamma)$.

We now claim that $x(\gamma) \in K$. If not, then there exist $y \in X$ and $C \in \mathcal{D}$ such that, for all $j \in C$, we have $u_{j}(y)>u_{j}(x(\gamma))$. From the above argument, it follows that $v_{j}=u_{j}(x(\gamma))$ for all $j \in N$, and so we have $u_{j}(y)>\left(1-\delta^{k}\right) u_{j}(q)+\delta^{k} v_{j}\left(\sigma^{k}\right)$ for all $j \in C$ and for high enough $k$. Thus, $y \in A^{k}$ for high enough $k$. Let $w_{j}^{k}(j)=\int u_{j} \pi_{j}^{k}$ be $j$ 's expected payoff when she proposes. This converges to $u_{j}(x(\gamma))$, since $\pi_{j}^{k}$ converges to the point mass on $x(\gamma)$, and sequential rationality then implies $w_{j}^{k}(j) \geq u_{j}(y)>$ $u_{j}(x(\gamma))=\lim w_{j}^{k}(j)$ for all $j \in C$ and for high enough $k$, a contradiction. Thus, we conclude that $x(\gamma) \in K$.

Finally, if not $D\left(A^{k}, K\right) \rightarrow 0$, then there must exist $\epsilon>0$ and a subsequence (also indexed by $k$ ) such that $D\left(A^{k}, K\right) \geq \epsilon$ for all $k$. We have shown that there is a further subsequence (also indexed by $k$ ) such that each $\pi_{i}^{k}$ converges to the point mass 
on some $x \in K$. Then $d\left(A^{k}, x\right) \geq \epsilon$ implies that there exist $y^{k} \in A^{k}$ and $C^{k} \in \mathcal{D}$ such that $d\left(y^{k}, x\right)>\epsilon / 2$ and $y^{k} \in A_{C^{k}}^{k}$. Going to a subsequence (still indexed by $k$ ) if needed, we may assume $C^{k}=C$ for all $k$ and $y^{k} \rightarrow y$. Thus, we have $u_{i}\left(y^{k}\right) \geq$ $\left(1-\delta^{k}\right) u_{i}(q)+\delta^{k} v_{i}\left(\sigma^{k}\right)$ for all $i \in C$ and all $k$, and continuity then implies $u_{i}(y) \geq u_{i}(\bar{x})$ for all $i \in C$. Since $d(y, x) \geq \epsilon / 2$, we have $y \neq x$, and by LSWP, there exists $z \in X$ such that $u_{i}(z)>u_{i}(x)$ for all $i \in C$. Thus, for high enough $k$, we have $u_{i}(z)>\left(1-\delta^{k}\right) u_{i}(q)+\delta^{k} v_{i}\left(\sigma^{k}\right)$ for all $i \in C$, which implies $z \in A^{k}$ for high enough $k$. Sequential rationality then implies $w_{i}^{k}(i) \geq u_{i}(z)>u_{i}(x)=\lim w_{i}^{k}(i)$ for high enough $k$, a contradiction. Therefore, $D\left(A^{k}, K\right)$ indeed converges to zero.

\section{REFERENCES}

Aliprantis, Charalambos, and Kim Border. 1994. Infinite Dimensional Analysis: A Hitchhiker's Guide. Berlin: Springer-Verlag.

Austen-Smith, David, and Jeffrey Banks. 1999. Positive Political Theory I: Collective Preference. Ann Arbor: University of Michigan Press.

Banks, Jeffrey. 1995. "Singularity Theory and Core Existence in the Spatial Model." Fournal of Mathematical Economics 24: 523-36.

Banks, Jeffrey S., and John Duggan. 1999. “A Bargaining Model of Collective Choice.” Caltech Social Science Working Paper no. 1053, California Institute of Technology.

Banks, Jeffrey, and John Duggan. 2000. “A Bargaining Model of Collective Choice.” American Political Science Reviem 94: 73-88.

Banks, Jeffrey, and John Duggan. 2005a. "A Social Choice Lemma on Voting Over Lotteries.” Social Choice and Welfare. Forthcoming.

Banks, Jeffrey, and John Duggan. 2005b. "A General Bargaining Model of Legislative Policy-making.” Mimeo.

Baron, David. 1991. "A Spatial Bargaining Theory of Government Formation in Parliamentary Systems." American Political Science Reviem 85: 137-64.

Baron, David. 1996. "A Dynamic Theory of Collective Goods Programs." American Political Science Reviem 90: 316-30.

Baron, David, and John Ferejohn. 1989. "Bargaining in Legislatures.” American Political Science Reviem 83: 1181-206.

Baron, David, and Michael Herron. 2003. "A Dynamic Model of Multidimensional Collective Choice." In Computational Models in Political Economy, ed. Ken Kollman, John Miller, and Scott Page. Cambridge, MA: MIT Press, 13-48.

Baron, David, and Ehud Kalai. 1993. "The Simplest Equilibrium of a Majority Rule Division Game.” Fournal of Economic Theory 61: 290-301.

Binmore, Kenneth. 1987. "Perfect Equilibria in Bargaining Models." In The Economics of Bargaining, ed. Kenneth Binmore and Partha Dasgupta. Oxford: Basil Blackwell, 77-105.

Black, Duncan. 1958. The Theory of Committees and Elections. Cambridge: Cambridge University Press.

Calvert, Randall, and Nathan Dietz. 2005. "Legislative Coalitions in a Bargaining Model with Externalities." In Social Choice and Strategic Decisions: Essays in Honor of Feffrey S. Banks, ed. David Austen-Smith and John Duggan. New York: Springer, 13-48.

Cho, Seok-ju, and John Duggan. 2003. "Uniqueness of Stationary Equilibria in a One-dimensional Model of Bargaining." Fournal of Economic Theory 113: 118-30.

Cox, Gary. 1984. "Non-collegial Simple Games and the Nowhere Denseness of the Set of Preference Profiles having a Core." Social Choice and Welfare 1: 159-64.

Diermeier, Daniel, Hulya Eraslan, and Antonio Merlo. 2002. "Coalition Governments and Comparative Constitutional Design.” European Economic Reviem 46: 893-907.

Eraslan, Hulya. 2002a. "Corporate Bankruptcy Reorganizations: Estimates from a Bargaining Model." Mimeo, University of Pennsylvania. 
Eraslan, Hulya. 2002b. "Uniqueness of Stationary Equilibrium Payoffs in the Baron-Ferejohn Model." Fournal of Economic Theory 103: 11-30.

Eraslan, Hulya, and Antonio Merlo. 2002. "Majority Rule in a Stochastic Model of Bargaining.” Fournal of Economic Theory 103: 31-48.

Harrington, Joseph. 1989. "The Advantageous Nature of Risk Aversion in a Three-player Bargaining Game where Acceptance of a Proposal Requires a Simple Majority." Economics Letters 30: 195-200.

Harrington, Joseph. 1990a. "The Power of the Proposal Maker in a Model of Endogenous Agenda Formation." Public Choice 64: 1-20.

Harrington, Joseph. 1990b. "The Role of Risk Preferences in Bargaining when Acceptance of a Proposal Requires less than Unanimous Approval." Fournal of Risk and Uncertainty 3: 135-54.

Hinich, Melvin, and Michael Munger. 1997. Analytical Politics. Cambridge: Cambridge University Press.

Jackson, Matthew, and Boaze Moselle. 2002. "Coalition and Party Formation in a Legislative Voting Game." Fournal of Economic Theory 103: 49-87.

Krehbiel, Keith. 1996. "Institutional and Partisan Sources of Gridlock: A Theory of Divided and Unified Government.” Fournal of Theoretical Politics 8: 7-40.

Krehbiel, Keith. 1998. Pivotal Politics: A Theory of U.S. Lammaking. Chicago: University of Chicago Press.

Le Breton, Michel. 1987. “On the Core of Voting Games.” Social Choice and Welfare 4: 295-305.

McCarty, Nolan. 2000. "Proposal Rights, Veto Rights, and Political Bargaining." American fournal of Political Science 44: 506-22.

McKelvey, Richard, and Norman Schofield. 1987. "Generalized Symmetry Conditions at a Core Point." Econometrica 55: 923-34.

Merlo, Antonio. 1997. "Bargaining Over Governments in a Stochastic Environment." Fournal of Political Economy 105: 101-31.

Merlo, Antonio, and Charles Wilson. 1995. "A Stochastic Model of Sequential Bargaining with Complete Information." Econometrica 63: 371-99.

Plott, Charles. 1967. "A Notion of Equilibrium and its Possibility under Majority Rule." American Economic Reviem 57: 787-806.

Powell, Robert. 2002. "Bargaining Theory and International Conflict." Annual Reviem of Political Science 5: 1-30.

Romer, Thomas, and Howard Rosenthal. 1978a. "Bureaucrats versus Voters: On the Political Economy of Resource Allocation by Direct Democracy." Quarterly Fournal of Economics 93: 563-87.

Romer, Thomas, and Howard Rosenthal. 1978b. "Political Resource Allocation, Controlled Agendas, and the Status Quo." Public Choice 33: 27-44.

Rubinstein, Ariel. 1979. "A Note on the Nowhere Denseness of Societies having an Equilibrium under Majority Rule." Econometrica 47: 511-14.

Rubinstein, Ariel. 1982. "Perfect Equilibrium in a Bargaining Model." Econometrica 50: 97-109.

Rubinstein, Ariel. 1985. "A Bargaining Model with Incomplete Information about Time Preferences." Econometrica 54: 1151-72.

Saari, Donald. 1997. "The Generic Existence of a Core for q-Rules." Economic Theory 9: 219-60.

Schofield, Norman. 1983. "Generic Instability of Majority Rule." Reviem of Economic Studies 50: 695-705.

Winter, Eyal. 1996. "Voting and Vetoing." American Political Science Reviem 90: 813-23. 
